\title{
(U) economics
}

One-seller assignment markets with multiunit demands

Francisco Robles

Marina Núñez 


\title{
One-seller assignment markets with multi-unit demands
}

\begin{abstract}
We consider one-seller assignment markets with multi-unit demands and prove that the associated game is buyers-submodular. Therefore the core is non-empty and it has a lattice structure which contains the allocation where every buyer receives his marginal contribution. We prove that in this kind of market, every pairwise-stable outcome is associated to a competitive equilibrium and vice versa. We study conditions under which the buyers-optimal and the seller-optimal core allocations are competitive equilibrium payoff vectors. Moreover, we characterize the markets for which the core coincidences with the set of competitive equilibria payoff vectors. When agents behave strategically, we introduce a procedure that implements the buyers-optimal core allocation as the unique subgame perfect Nash equilibrium outcome.
\end{abstract}

JEL Codes: C71, C78.

Keywords: Many-to-many assignment markets, Core, Pairwise-stability, Competitive equilibrium.

Francisco Robles

Facultat d'Economia i Empresa

Universitat de Barcelona

Marina Núñez

Facultat d'Economia i Empresa

Universitat de Barcelona

Acknowledgements: The authors acknowledge the support by research grant ECO2011-22765 (Spanish Ministry of Science and Innovation and FEDER) and 2013SGR40 (Government of Catalonia). The first author also acknowledges the support of the FPU program (Spanish Ministry of Education). 


\section{Introduction}

We study markets with several buyers and only one seller. The seller owns many indivisible and potentially different objects on sale. Being heterogeneous, the objects are of the same type: for instance different houses or different tasks. On the other side of the market, each buyer has a nonnegative valuation for each object and a desire to acquire a certain number of objects. This number is known as the capacity of the buyer. We assume buyers value packages of objects additively. Utility is identified with money and side-payments are allowed.

This market is a particular case of the one considered in Jaume et al. (2012), where there are several sellers, each with a set of heterogeneous objects on sale. It is also a particular case of the package auction of Ausubel and Milgrom (2002), where there is also only one seller, but buyers may not value packages additively. A related situation, also with only one seller owning many objects on sale but buyers with unitary demands, was analyzed in Camiña (2006).

Two-sided markets with transferable utility are first considered from the viewpoint of coalitional games in the assignment game (Shapley and Shubik, 1972). In this model, there are two disjoint finite sets of agents: a set of sellers, each supplying one unit of an indivisible good, and a set of buyers, each of them demanding one unit of the good. Each buyer may valuate differently the object of each seller and, from these valuations, we summarize in the valuation matrix the potential worth of each buyer-seller partnership. The worth of an arbitrary coalition of buyers and sellers is the maximum profit that can be achieved by optimally matching buyers and sellers inside the coalition.

In the assignment game, the core is non-empty and coincides with the set of solutions of the linear program dual to the optimal assignment problem. As a consequence, the core coincides with those efficient payoff vectors that satisfy pairwise-stability, that is to say, no pair of a buyer and a seller would be better off by breaking their optimal partnership in any optimal matching and being matched together (Shapley and Shubik, 1972). Moreover, the set of competitive equilibria payoff vectors are in one-to-one correspondence with the set of solutions of the dual linear assignment problem (Gale, 1960). Hence, this is another characterization of the core of the assignment game in terms of the competitive prices. Even more, the core of the assignment game is a complete lattice with two particular core elements, one of them optimal for all buyers and the other one optimal for all sellers.

When the assumptions of the classical assignment model are relaxed, the coincidence between the core, the set of pairwise-stable outcomes, the set of competitive equilibria payoff vectors and the set of solutions of the dual optimal assignment problem does not hold in general. The same happens with the lattice structure of some of these sets.

The first generalization of the classical assignment game considers that each seller owns several units of different goods and can be matched to as many buyers as allowed by the seller's capacity. On the other hand, buyers' demand is still unitary (Kaneko, 1976). In this many-to-one assignment model (many agents on the buyers' side can be matched to a same agent on the sellers' side), the set of payoff vectors associated to the competitive equilibria is included in the core, which guarantees the non-emptiness of the core. Nevertheless, this inclusion may be strict.

Other generalizations of the assignment game are known as many-to-many markets 
since both buyers and sellers may have capacities greater than one: the capacity of a buyer is the number of objects he desires to buy and the capacity of a seller is the number of identical objects she offers on sale (Thompson, 1980). A matching describes a set of partnerships between buyers and sellers within their quotes, and allows for multiunit trade within a same buyer-seller pair. This two-sided market can be considered to represent a job market between heterogeneous firms and workers, when the objects on sale are units of labour as in Sotomayor (2002). Optimal matchings for the many-tomany assignment market are obtained by solving a linear transportation problem. The core of this game is always non-empty but it remains an open problem whether in this setting an optimal core element for each side of the market does exist, although it is known that a worst core element for each side of the market needs not exist.

A more encompassing many-to-many assignment model is the one with heterogeneous goods and multi-unit demands of Jaume et al. (2012), where several sellers own several units of potentially different objects. In these markets, as in the many-to-one markets of Kaneko (1976), the set of solutions of the dual transportation problem coincides with the set of competitive equilibrium payoff vectors and it is strictly included in the core. However, let us point out that the definition of competitive equilibrium in Jaume et al. (2012) assumes that buyers demand as many copies of their preferred object as their capacities allow, being the prices given. Compared to that, in a many-to-many assignment game in which the goods owned by a seller are homogeneous, Sotomayor (2013) defines competitive equilibria by means of a demand in which buyers maximize the utility of the packages they can buy given prices and their capacities. ${ }^{1}$

In the present paper, where we have only one seller with heterogeneous goods and several buyers with multi-unit demands, we first prove that the corresponding coalitional function is buyer-submodular. Then, as a consequence of Ausubel and Milgrom (2002), we deduce: a) an easy description of the core as the non-empty set of efficient payoff vectors where each buyer gets a non-negative payoff bounded by his marginal contribution to the whole market; b) the core is endowed with a lattice structure by the partial order defined from the point of view of buyers, and c) the existence of a core element that is optimal for each side of the market. Moreover, as in the assignment game, in the buyers-optimal core allocation each buyer is paid his marginal contribution, the Vickrey payoff (Vickrey, 1961).

The set of (discriminatory) competitive equilibria of the one-seller assignment game does not in general coincide neither with the core nor with the set of solutions of the dual transportation problem. We first prove that the set of payoff vectors associated to the pairwise-stable outcomes coincides with the set of those associated to the competitive equilibria. Secondly, we give conditions so that the buyers-optimal core allocation or the seller-optimal core allocation are payoff vectors associated to some competitive equilibrium. Moreover, we provide conditions on the valuation matrix under which the set of competitive equilibria payoff vectors coincide with the core.

\footnotetext{
${ }^{1}$ Sotomayor (2013) differentiates between the two definitions of competitive equilibrium. In the one in Jaume et al. (2012) demands are non-discriminatory, since each buyer gets the same utility from all objects in his demanded sets. The demand in Sotomayor (2013) is discriminatory since in a demanded package the buyer may obtain different utilities from the different objects that form the package. Recently, Arribillaga et al. (2013) consider a discriminatory competitive equilibrium in assignment markets where sellers own heterogeneous objects.
} 
In the literature of assignment markets, it is usual to implement some outstanding core outcomes by means of the Nash equilibrium of some convenient game in strategic form. Demange et al. (1986) reach the buyers-optimal core allocation of the one-to-one assignment game by means of a multi-item auction in which buyers compete for the objects under certain rules. Likewise, under the assumption of complete information, Pérez-Castrillo and Sotomayor (2002) implement the sellers-optimal core allocation of the assignment game by means of a mechanism in which both buyers and sellers play a strategic role.

For the one-seller assignment market with multi-unit demands, and also under the assumption of complete information, we propose a mechanism to implement, as the unique subgame perfect Nash equilibrium outcome, the buyers-optimal core allocation. This mechanism works as follows. Simultaneously, each buyer declares to the seller a maximal subset of pairs formed by a package and its price, being each buyer indifferent among all the pairs in his declaration. In a second step, the seller selects a matching of buyers to packages of objects that is compatible with the requirements declared by the buyers. A buyer that gets a package pays the price he announced.

The paper is organized as follows. In the next section, preliminaries are addressed. In Section 3, we prove the buyers-submodularity property of the coalitional function, and also characterize convexity. In Section 4, we consider the set of competitive equilibria and pairwise-stable outcomes, and their relationship with the core. Section 5 is devoted to the mechanism that implements, as the unique subgame perfect Nash equilibrium outcome, the buyers-optimal core allocation of one-seller assignment markets. Section 6 concludes.

\section{The model and some preliminaries}

The one-seller assignment market with multi-unit demands consists of $(M,\{0\}, Q, A, r)$. The finite set of buyers is $M=\{1, \ldots, m\}$ and the unique seller is denoted by 0 . The seller owns a finite set $Q$ of objects. These objects are indivisible and heterogeneous, but of a similar type, let us say different houses or different part-time jobs.

Each buyer-object pair $(i, j) \in M \times Q$ has a potential gain $a_{i j} \in \mathbb{R}_{+}$, interpreted as the valuation of object $j$ by buyer $i$. The valuation matrix, denoted by $A=$ $\left(a_{i j}\right)_{(i, j) \in M \times Q}$, captures each potential gain among all buyer-object pairs.

Each buyer $i \in M$ can acquire $r_{i} \in \mathbb{N}$ objects. We say that buyer $i$ has a unitary demand if $r_{i}=1$ and a multi-unit demand if $r_{i}>1$. The vector $r=\left(r_{i}\right)_{i \in M} \in \mathbb{N}^{M}$ indicates buyers' capacities. We assume that the seller owns some copies of a dummy object, as many as the sum of all buyers' capacities, $\sum_{i \in M} r_{i}$. With some abuse of notation, each copy of this dummy object is denoted by $j_{0}$ and each buyer values it at zero. We denote by $2_{r_{i}}^{Q}=\left\{R \subseteq Q ;|R|=r_{i}\right\}$ the set of allowable packages of objects for a buyer $i \in M$ where $|R|$ denotes the cardinality of the set $R$. We denote by $A_{\mid S \times Q}$ the valuation matrix restricted to $S \times Q$, where $S \subseteq M$. Besides, let $r_{\mid S}$ be the vector of capacities restricted to $S \subseteq M$.

A feasible matching $\mu$ for a market $\left(S,\{0\}, Q, A_{\mid S \times Q}, r_{\mid S}\right)$, between $S \subseteq M$ and $Q$, is a subset of $S \times Q$ such that each $j \in Q$ belongs to at most one pair and each $i \in S$ 
belongs to exactly $r_{i}$ pairs. Notice that it is possible to match any buyer with dummy objects to complete his capacity. We denote by $\mathcal{M}(S, Q)$ the set of feasible matchings between $S \subseteq M$ and $Q$, and $\mu(S)$ is the set of objects matched by $\mu$ to all buyers in $S$, and when $S=\{i\}$ we simply write $\mu(i)$. Moreover, we denote by $\mu^{-1}(j)$ the buyer matched to object $j \in Q$ by matching $\mu$.

We assume buyers valuate packages of objects additively. That is, if buyer $i$ is assigned a package $\mu(i) \subseteq Q$ by some $\mu \in \mathcal{M}(M, Q)$, then his valuation of this package is $\sum_{j \in \mu(i)} a_{i j}$.

A feasible matching $\mu$ is optimal for a market $\left(S,\{0\}, Q, A_{\mid S \times Q}, r_{\mid S}\right)$ if

$$
\sum_{(i, j) \in \mu} a_{i j} \geq \sum_{(i, j) \in \mu^{\prime}} a_{i j} \quad \text { for all } \mu^{\prime} \in \mathcal{M}(S, Q) .
$$

We denote by $\mathcal{M}_{A}(S, Q)$ the set of optimal matchings for this market.

Let us introduce a coalitional game with transferable utility (a game) ${ }^{2}$. Consider a one-seller assignment market with multi-unit demands $(M,\{0\}, Q, A, r)$. The one-seller assignment game related to $(M,\{0\}, Q, A, r)$ is denoted by $\left(M \cup\{0\}, v_{A}\right)$. The worth of each coalition $T \subseteq M \cup\{0\}$ is given by

$$
v_{A}(T)= \begin{cases}\max _{\mu \in \mathcal{M}(T \backslash\{0\}, Q)}\left\{\sum_{(i, j) \in \mu} a_{i j}\right\} & \text { if }\{0\} \varsubsetneqq T, \\ 0 & \text { if } T \cap\{0\}=\emptyset \text { or } T=\{0\} .\end{cases}
$$

Now, we define competitive equilibrium for one-seller assignment markets with multiunit demands. First, let us introduce some notions. A feasible price vector $p=\left(p_{j}\right)_{j \in Q} \in$ $\mathbb{R}_{+}^{Q}$ for $(M,\{0\}, Q, A, r)$ consists of one price for each object, with a price of zero for each dummy object. For each $p \in \mathbb{R}_{+}^{Q}$, we denote by $D_{i}(p) \subseteq 2_{r_{i}}^{Q}$ the demand set of buyer $i$ at level prices $p$, that is

$$
D_{i}(p)=\left\{R \in 2_{r_{i}}^{Q} \mid \sum_{j \in R}\left(a_{i j}-p_{j}\right) \geq \sum_{j \in R^{\prime}}\left(a_{i j}-p_{j}\right) \text { for all } R^{\prime} \in 2_{r_{i}}^{Q}\right\} .
$$

Thus, when a package belongs to the demand set of a buyer at prices $p$, he weakly prefers to buy this set than every other package at $p$. The demand set of any buyer is never empty, since even at sufficiently high prices, the demand set can be formed by as many dummy objects as needed.

\footnotetext{
${ }^{2} \mathrm{~A}$ coalitional game with transferable utility is a pair $(N, v)$ where $N$ is a finite set of players and $v$ is the coalitional function defined on the set $2^{N}$ formed by all coalitions of $N$. Thereby, to each $S \subseteq N$, function $v$ assigns a real value $v(S)$, which is interpreted as the worth generated by the players in $S$ on their own; furthermore, $v(\emptyset)=0$. A payoff vector is $x \in \mathbb{R}^{N}$. For every $S \subseteq N$ we write $x(S)=\sum_{i \in S} x_{i}$ to express the payoff to coalition $S$, where $x(\emptyset)=0$. A payoff vector $x$ satisfies efficiency if $x(N)=v(N)$ and $x$ satisfies individual rationality if $x_{i} \geq v(\{i\})$ for all $i \in N$. A payoff vector $x$ belongs to the imputation set $I(v)$, if it satisfies individual rationality and efficiency. The core of a game is defined by $C(v)=\left\{x \in \mathbb{R}^{N} \mid x(N)=v(N)\right.$ and $x(S) \geq v(S)$ for all $\left.S \subset N\right\}$. Finally, a game $(N, v)$ is monotonic if $v(S) \geq v(T)$ for all $T \subseteq S \subseteq N$. A game $(N, v)$ is convex if $v(T \cup\{i\})+v(T) \leq v(S \cup\{i\})+v(S)$ for all $T \subseteq S \subseteq N \backslash\{i\}$ and all $i \in N$.
} 
Definition 2.1. Let $(M,\{0\}, Q, A, r)$ be a one-seller assignment market with multi-unit demands. A pair formed by a feasible price vector $p \in \mathbb{R}_{+}^{Q}$ and a feasible matching $\mu \in \mathcal{M}(M, Q)$, denoted by $(p, \mu)$, is a competitive equilibrium if the following two conditions hold:

C.1 For all $i \in M, \mu(i) \in D_{i}(p)$,

C.2 For all $j \in Q \backslash \mu(M), p_{j}=0$.

If a pair $(p, \mu)$ is a competitive equilibrium, we say that $p$ is a competitive equilibrium price vector. In a competitive equilibrium, every buyer maximizes his utility given the prices for the objects. This notion of competitive equilibrium for buyers with capacities is considered in Sotomayor (2013) and Arribillaga et al. (2013).

Definition 2.2. Let $(M,\{0\}, Q, A, r)$ be a one-seller assignment market with multi-unit demands. Given a feasible price vector $p \in \mathbb{R}_{+}^{Q}$ and a feasible matching $\mu \in \mathcal{M}(M, Q)$, the payoff vector associated to $(p, \mu)$ is $(U(p, \mu), V(p, \mu)) \in \mathbb{R}^{M} \times \mathbb{R}$ defined as follows:

$$
\begin{aligned}
U_{i}(p, \mu) & =\sum_{j \in \mu(i)}\left(a_{i j}-p_{j}\right) \quad \text { for each } i \in M, \text { and } \\
V(p, \mu) & =\sum_{j \in Q} p_{j} \quad \text { for the seller. }
\end{aligned}
$$

The following consequences regarding the set of competitive equilibria follow easily for one-seller assignment markets with multi-unit demands. We omit the proof since it can also be derived from results on a more general market in Arribillaga et al. (2013).

R1. The set of competitive equilibria is the cartesian product of the set of competitive equilibria price vectors and the set of optimal matchings.

R2. The payoff vector $(U(p, \mu), V(p, \mu))$ associated to any competitive equilibrium $(p, \mu)$ belongs to the core of the associated game.

R3. The set of competitive equilibria of the market is non-empty.

Notice that the seller plays no role in the definition of the competitive equilibrium. That is, $(p, \mu)$ is a competitive equilibrium for the one-seller assignment market with multi-unit demands $(M,\{0\}, Q, A, r)$ if and only if it is a competitive equilibrium for the market where each object in $Q$ is identified with a different seller. Then, as a consequence of Sotomayor (2007), the set of competitive equilibrium prices is a complete lattice with the usual order: $p \geq p^{\prime}$ if $p_{j} \geq p_{j}^{\prime}$ for all $j \in Q$. This means that if $p, p^{\prime} \in \mathbb{R}_{+}^{Q}$ are competitive prices then $p \vee p^{\prime}$ and $p \wedge p^{\prime}$ are also competitive prices, where $\left(p \vee p^{\prime}\right)_{j}=\max \left\{p_{j}, p_{j}^{\prime}\right\}$ and $\left(p \wedge p^{\prime}\right)_{j}=\min \left\{p_{j}, p_{j}^{\prime}\right\}$ for all $j \in Q$.

To finish this section, let us introduce the buyers-submodular condition as in Ausubel and Milgrom (2002). This condition means that the marginal contribution of a buyer to a coalition containing the seller decreases as the coalition grows larger. A game 
( $M \cup\{0\}, v)$ is buyers-submodular if any of the three following equivalent conditions holds:

(i) $\quad v((T \cup\{0\}) \cup\{i\})-v(T \cup\{0\}) \geq v((S \cup\{0\}) \cup\{i\})-v(S \cup\{0\})$

for all $T \subseteq S \subseteq M \backslash\{i\}$ and all $i \in M$.

(ii)

$$
v(S \cup\{0\})-v(T \cup\{0\}) \geq \sum_{i \in S \backslash T}(v(S \cup\{0\})-v((S \backslash\{i\}) \cup\{0\}))
$$

for all $T \subseteq S \subseteq M$.

(iii) $\quad v\left(S_{1} \cup\{0\}\right)+v\left(S_{2} \cup\{0\}\right) \geq v\left(\left(S_{1} \cup S_{2}\right) \cup\{0\}\right)+v\left(\left(S_{1} \cap S_{2}\right) \cup\{0\}\right)$

for all $S_{1}, S_{2} \subseteq M$.

In Ausubel and Milgrom (2002), it is proved that if a game $(M \cup\{0\}, v)$ satisfies the buyers-submodular condition, the core is easily determined by

$$
C(v)=\left\{(U, V) \in \mathbb{R}^{M} \times \mathbb{R} \mid \sum_{i \in M} U_{i}+V=v(M \cup\{0\}), 0 \leq U_{i} \leq M_{i}^{v} \text { for all } i \in M\right\},
$$

where $M_{i}^{v}=v(M \cup\{0\})-v((M \cup\{0\}) \backslash\{i\})$ denotes the marginal contribution of buyer $i \in M$ to the grand coalition.

\section{One-seller assignment game: the core}

In order to describe the core of the one-seller assignment game with multi-unit demands, we first show that it satisfies the buyers-submodularity condition.

Theorem 3.1. Let $(M,\{0\}, Q, A, r)$ be a one-seller assignment market with multi-unit demands and $\left(M \cup\{0\}, v_{A}\right)$ be its related one-seller assignment game. Then $\left(M \cup\{0\}, v_{A}\right)$ is buyers-submodular.

Proof. First, consider the unitary-demands case $\left(r_{i}=1\right.$ for all $\left.i \in M\right)$. Let $\left(M \cup\{0\}, v_{A}\right)$ be the one-seller assignment game with unitary demands.

We deduce from Theorem 1 in Shapley (1962), that for all $i, i^{\prime} \in M$ and all $S \subseteq$ $M \backslash\left\{i, i^{\prime}\right\}$

$$
v_{A}((S \cup\{0\}) \cup\{i\})-v_{A}(S \cup\{0\}) \geq v_{A}\left((S \cup\{0\}) \cup\left\{i, i^{\prime}\right\}\right)-v_{A}\left((S \cup\{0\}) \cup\left\{i^{\prime}\right\}\right),
$$

and by repeatedly applying this, we obtain that $\left(M \cup\{0\}, v_{A}\right)$ satisfies condition $(i)$.

Now, consider a one-seller assignment market with multi-unit demands. We prove that $\left(M \cup\{0\}, v_{A}\right)$ is buyers-submodular, that is

$$
v_{A}((T \cup\{0\}) \cup\{i\})-v_{A}(T \cup\{0\}) \geq v_{A}((S \cup\{0\}) \cup\{i\})-v_{A}(S \cup\{0\}),
$$

for all $T \subseteq S \subseteq M \backslash\{i\}$ and all $i \in M$. Define a related market in which each buyer $i \in M$ is replicated $r_{i}$ times. Denote by $i(s)$ the $s$-th copy of $i$ (each copy has capacity one) and by $\widetilde{M}$ the new set of buyers formed by replicating all buyers in $M$. Define the 
valuation matrix $\widetilde{A}=\left(a_{i(s) j}\right)_{(i(s), j) \in \widetilde{M} \times Q}$ by $a_{i(s) j}=a_{i j}$ for all $(i, j) \in M \times Q$ and all $s \in\left\{1, \ldots, r_{i}\right\}$. In this way, we obtain $(\widetilde{M},\{0\}, Q, \widetilde{A}, \widetilde{r})$, a one-seller assignment market with unitary demands. Notice that $\left(M \cup\{0\}, v_{A}\right)$ and $\left(\widetilde{M} \cup\{0\}, v_{\widetilde{A}}\right)$ are related:

$$
v_{A}(S \cup\{0\})=v_{\widetilde{A}}(\widetilde{S} \cup\{0\}) \text { for all } S \subseteq M,
$$

where $\widetilde{S}$ is formed by replicating all buyers in $S$. Then, inequality (6) is equivalent to

$$
\begin{aligned}
v_{\widetilde{A}}\left((\widetilde{T} \cup\{0\}) \cup\left\{i(1), \ldots, i\left(r_{i}\right)\right\}\right) & -v_{\widetilde{A}}(\widetilde{T} \cup\{0\}) \\
& \geq v_{\widetilde{A}}\left((\widetilde{S} \cup\{0\}) \cup\left\{i(1), \ldots, i\left(r_{i}\right)\right\}\right)-v_{\widetilde{A}}(\widetilde{S} \cup\{0\}),
\end{aligned}
$$

where $\widetilde{T}, \widetilde{S}$ and $i(1), \ldots, i\left(r_{i}\right)$ are obtained by replicating all buyers in $T, S$ and $\{i\}$, respectively. Define $S_{1}=\widetilde{T} \cup\left\{i(1), \ldots, i\left(r_{i}\right)\right\}, S_{2}=\widetilde{S}$ and notice that $S_{1} \cup S_{2}=\widetilde{S} \cup$ $\left\{i(1), \ldots, i\left(r_{i}\right)\right\}$ and $S_{1} \cap S_{2}=\widetilde{T}$. Since when demands are unitary the game is buyerssubmodular, $\left(\widetilde{M} \cup\{0\}, v_{\widetilde{A}}\right)$ satisfies (2), which is equivalent to (4). Then

$$
v_{\widetilde{A}}\left(S_{1} \cup\{0\}\right)+v_{\widetilde{A}}\left(S_{2} \cup\{0\}\right) \geq v_{\widetilde{A}}\left(\left(S_{1} \cup S_{2}\right) \cup\{0\}\right)+v_{\widetilde{A}}\left(\left(S_{1} \cap S_{2}\right) \cup\{0\}\right),
$$

and by reordering terms we obtain (8). Therefore (6) holds. Hence $\left(M \cup\{0\}, v_{A}\right)$ is buyers-submodular.

From Theorem 3.1, the one-seller assignment game is buyers-submodular, and by Ausubel and Milgrom (2002), its core is non-empty and can be described as in (5). That is: the set of nonnegative efficient payoff vectors where each buyer gets a payoff not exceeding his marginal contribution. Furthermore, the core is a lattice with respect to the usual order defined on buyers' payoffs. As a consequence, we can guarantee the existence of one optimal core allocation for each side of the market. In the buyersoptimal core allocation $(\bar{U}, \underline{V}) \in \mathbb{R}^{M} \times \mathbb{R}$, each buyer gets his marginal contribution, that is, $\bar{U}_{i}=M_{i}^{v_{A}}$ for all $i \in M$ and $\underline{V}=v_{A}(M \cup\{0\})-\sum_{i \in M} M_{i}^{v_{A}}$. On the other hand, in the seller-optimal core allocation $(\underline{U}, \bar{V}) \in \mathbb{R}^{M} \times \mathbb{R}$, each buyer $i \in M$ gets $\underline{U}_{i}=0$ and $\bar{V}=v_{A}(M \cup\{0\})$. Thus, the core of the one-seller assignment game has an optimal core allocation for each market sector as it happens in the classical assignment game. This is not known to be true for other many-to-many assignment models (see e.g. Sotomayor, 2002).

Now, we turn to study what happens if there is a special optimal matching in which each buyer $i \in M$ is matched to his most valued $r_{i}$ objects. In this case, we obtain that the one-seller assignment game is convex. Equivalently, under the convexity assumption, the worth of each coalition formed by a group of buyers and the seller, is obtained by adding what the seller can generate with each of these buyers.

Proposition 3.2. Let $(M,\{0\}, Q, A, r)$ be a one-seller assignment market with multiunit demands and $\left(M \cup\{0\}, v_{A}\right)$ be its related one-seller assignment game. Then the following statements are equivalent:

1. $\left(M \cup\{0\}, v_{A}\right)$ is a convex game, 
2. $v_{A}(S \cup\{0\})=\sum_{i \in S} v_{A}(\{i, 0\})$ for all $S \subseteq M$.

Proof. First, we prove $1 \Rightarrow 2$. Assume $\left(M \cup\{0\}, v_{A}\right)$ is a convex game, and let $S=$ $\left\{i_{1}, \ldots, i_{s}\right\} \subseteq M$. Notice that

$$
\begin{aligned}
v_{A}(S \cup\{0\})-v_{A}(\{0\}) & =\sum_{l=2}^{s}\left(v_{A}\left(\left\{i_{1}, \ldots, i_{l}, 0\right\}\right)-v_{A}\left(\left\{i_{1}, \ldots, i_{l-1}, 0\right\}\right)\right) \\
& +v_{A}\left(\left\{i_{1}, 0\right\}\right)-v_{A}(\{0\}) .
\end{aligned}
$$

By the convexity of $\left(M \cup\{0\}, v_{A}\right)$, we obtain

$$
v_{A}(S \cup\{0\})-v_{A}(\{0\}) \geq \sum_{i \in S}\left(v_{A}(\{i, 0\})-v_{A}(\{0\})\right) .
$$

By the buyers-submodularity of $\left(M \cup\{0\}, v_{A}\right)$ applied to (9), we obtain

$$
v_{A}(S \cup\{0\})-v_{A}(\{0\}) \leq \sum_{i \in S}\left(v_{A}(\{i, 0\})-v_{A}(\{0\})\right) .
$$

Since $v_{A}(\{0\})=0$, by (10) and (11), we obtain statement 2 .

Now, $2 \Rightarrow 1$. We prove that for all $i^{\prime} \in M \cup\{0\}$ and all $T \subseteq S \subseteq(M \cup\{0\}) \backslash\left\{i^{\prime}\right\}$

$$
v_{A}\left(\left(T \cup\left\{i^{\prime}\right\}\right)\right)-v_{A}(T) \leq v_{A}\left(S \cup\left\{i^{\prime}\right\}\right)-v_{A}(S) .
$$

We consider several cases. If $0 \notin S$ and $i^{\prime} \in M$, condition (12) is trivially satisfied. If $0 \notin S$ and $i^{\prime}=0$, condition (12) is reduced to monotonicity and it is satisfied. If $0 \in S \backslash T$ and $i^{\prime} \in M$, condition (12) is also satisfied. Finally, we consider $0 \in T$ and $i^{\prime} \in M$. Take any $T \subseteq S \subseteq M \backslash\left\{i^{\prime}\right\}$, then

$$
\begin{aligned}
v_{A}\left(T \cup\left\{i^{\prime}\right\}\right)-v_{A}(T) & =\sum_{i \in(T \cap M) \cup\left\{i^{\prime}\right\}} v_{A}(\{i, 0\})-\sum_{i \in T \cap M} v_{A}(\{i, 0\}) \\
& =\sum_{i \in(S \cap M) \cup\left\{i^{\prime}\right\}} v_{A}(\{i, 0\})-\sum_{i \in S \cap M} v_{A}(\{i, 0\}) \\
& =v_{A}\left(S \cup\left\{i^{\prime}\right\}\right)-v_{A}(S),
\end{aligned}
$$

where first and third equalities come from our assumption. This completes the proof.

Notice that the above characterization of convexity for one-seller assignment games can be expressed by means of the valuation matrix. Indeed, such a game $\left(M \cup\{0\}, v_{A}\right)$ is convex if for any optimal matching $\mu \in \mathcal{M}_{A}(M, Q)$ and for all $i \in M$

$$
\sum_{j \in \mu(i)} a_{i j} \geq \sum_{j \in R} a_{i j} \text { for all } R \in 2_{r_{i}}^{Q} .
$$




\section{Competitive equilibria, pairwise-stability and the core}

Shapley and Shubik (1972) shows that the set of competitive outcomes coincides with the core in the classical assignment game. For several generalizations of the classical assignment game, those outcomes coming from a competitive equilibrium are core elements (Kaneko 1976; Camiña, 2006 and Sotomayor, 2013). However, the coincidence of the set of competitive equilibria payoff vectors with the core is not preserved in general.

The aim of this section is to analyze under which conditions the core of one-seller assignment game coincides with the set of competitive equilibria payoff vectors. To this end, we introduce the notion of pairwise-stability which has been widely studied in other many-to-many assignment markets (Sotomayor, 1992, 2002, 2007). It also appears in Sotomayor (2013) with the name of strong stability. This notion of pairwise-stability focuses on each trade and the profit arising from each individual purchase. Then, market stability by means of competitive equilibrium and pairwise-stability is analyzed.

Let us first define feasible configuration of profits and feasible outcomes.

Definition 4.1. Let $(M,\{0\}, Q, A, r)$ be a one-seller assignment market with multi-unit demands. Given a feasible matching $\mu \in \mathcal{M}(M, Q)$, a feasible configuration of profits compatible with $\mu$ is $\left(\left(u_{i j}\right)_{(i, j) \in \mu},\left(v_{j}\right)_{j \in Q}\right)=(u, v) \in \mathbb{R}^{b} \times \mathbb{R}^{Q}$, where $b=\sum_{i \in M} r_{i}$, such that:

1. $u_{i j}+v_{j}=a_{i j}, u_{i j} \geq 0, v_{j} \geq 0$ for all $(i, j) \in \mu$,

2. $v_{j}=0$ if $j \in Q \backslash \mu(M)$.

We can interpret $u_{i j}$ as the profit of buyer $i$ acquiring object $j$. Similarly, $v_{j}$ is the profit for the seller related to object $j$. If an object is not sold, then the seller receives a profit of zero from this object. Notice that $u_{i j}$ is not defined for $(i, j) \in M \times Q \backslash \mu$.

Definition 4.2. Let $(M,\{0\}, Q, A, r)$ be a one-seller assignment market with multi-unit demands. A feasible outcome for $(M,\{0\}, Q, A, r)$, denoted by $(u, v ; \mu)$, is formed by a feasible matching $\mu \in \mathcal{M}(M, Q)$ and a feasible configuration of profits $(u, v)$ compatible with $\mu$.

In the following, we define the pairwise-stable outcomes.

Definition 4.3. Let $(M,\{0\}, Q, A, r)$ be a one-seller assignment market with multi-unit demands. A feasible outcome $(u, v ; \mu)$ for $(M,\{0\}, Q, A, r)$, is a pairwise-stable outcome if for all $i \in M, u_{i j}+v_{k} \geq a_{i k}$ for all $j \in \mu(i)$ and all $k \in Q \backslash \mu(i)$.

A feasible outcome is pairwise-stable if there is no pair formed by a buyer $i \in M$ and an object $k \in Q$ that are not matched together by $\mu$ but, if they were (maybe after breaking a previous partnership), then the buyer would be better off and the seller would receive a higher profit from this object $k$. Notice that when a feasible outcome satisfies Definition 4.3, it is stable in strong sense since the agents do not regret any single partnership. Moreover, if we assume for a moment that each object were owned by a different seller, Definition 4.3 means that the feasible outcome is not 
blocked by any buyer-seller pair. Our next proposition asserts that, as it happens in the classical assignment market, each pairwise-stable outcome is associated with a competitive equilibrium and vice versa.

Proposition 4.4. Let $(M,\{0\}, Q, A, r)$ be a one-seller assignment market with multiunit demands. The feasible outcome $(u, v ; \mu)$ is a pairwise-stable outcome if and only if $(v, \mu)$ is a competitive equilibrium.

Proof. We first prove the "if" part. Let $(v, \mu)$ be a competitive equilibrium. Define $u \in \mathbb{R}^{b}$ by $u_{i j}=a_{i j}-v_{j}$ if $j \in \mu(i)$ for all $i \in M$. We see that $(u, v ; \mu)$ is a pairwisestable outcome. Assume on the contrary that $(u, v ; \mu)$ is not pairwise-stable, then there is some $j^{\prime} \in \mu(i)$ such that, $a_{i j^{\prime}}-v_{j^{\prime}}<a_{i k}-v_{k}$ for some $k \in Q \backslash \mu(i)$. Define $T=\left(\mu(i) \backslash\left\{j^{\prime}\right\}\right) \cup\{k\} \subseteq Q$. Then

$$
\sum_{j \in \mu(i)}\left(a_{i j}-v_{j}\right)<\sum_{j \in T}\left(a_{i j}-v_{j}\right)
$$

which implies $\mu(i) \notin D_{i}(v)$ and contradicts the fact that $(v, \mu)$ is a competitive equilibrium. Hence $(u, v ; \mu)$ is a pairwise-stable outcome.

Now, we prove the "only if" part. Given a pairwise-stable outcome $(u, v ; \mu)$ let us see that $(v, \mu)$ is a competitive equilibrium. Stability implies $u_{i j}=a_{i j}-v_{j} \geq a_{i k}-v_{k}$ for all $j \in \mu(i)$ and all $k \in Q \backslash \mu(i)$. We have that for any $T \in 2_{r_{i}}^{Q}, \sum_{j \in \mu(i)}\left(a_{i j}-v_{j}\right) \geq$ $\sum_{j \in T}\left(a_{i j}-v_{j}\right)$ and then $\mu(i) \in D_{i}(v)$ for each $i \in M$. Besides, by feasibility of $(u, v ; \mu)$, $v_{j}=0$ if $j \in Q \backslash \mu(M)$. Hence $(v, \mu)$ is a competitive equilibrium.

As a consequence of the coincidence stated in Proposition 4.4, we can easily describe the set of competitive equilibria outcomes by means of a set of finite linear equalities and inequalities, as shown in Example 4.5.

In the definition of competitive equilibrium, the seller plays no role and buyers maximize their utility, given the fixed prices for the objects. This is the reason why this coincidence between competitive equilibrium and pairwise-stability also holds for many-to-one assignment markets with several sellers where buyers may have different capacities and each seller only one object on sale. Nevertheless, this coincidence does not generally hold for many-to-many markets (see, e.g. Sotomayor, 2007).

However, as it is also the case for more general markets, the set of competitive equilibria payoff vectors of the one-seller assignment game may not coincide with the core. This is shown in the next example.

Example 4.5. Consider a market with only one seller and two buyers. The seller owns two objects on sale. Each buyer can acquire one object. For the purposes of this example, we show no dummy objects. That is, the market $(M,\{0\}, Q, A, r)$ is given by $M=\{1,2\}$, $Q=\left\{1^{\prime}, 2^{\prime}\right\}$ and $r=(1,1)$. The valuation matrix $A$ is the following

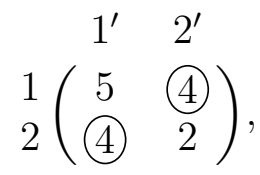


where the unique optimal matching is circled. Consider the one-seller assignment game $\left(M \cup\{0\}, v_{A}\right)$. The core is described by:

$$
C\left(v_{A}\right)=\left\{\begin{array}{l|l}
(U, V) \in \mathbb{R}^{2} \times \mathbb{R} & \begin{array}{l}
U_{1}+U_{2}+V=8 \\
0 \leq U_{1} \leq 4 \\
0 \leq U_{2} \leq 3 .
\end{array}
\end{array}\right\} .
$$

Now, we consider the set of competitive equilibria payoff vectors. Take the unique optimal matching $\mu$, where $\mu(1)=\left\{2^{\prime}\right\}$ and $\mu(2)=\left\{1^{\prime}\right\}$. By Proposition 4.4, we know that any competitive equilibrium $(v, \mu)$, and its related stable outcome $(u, v ; \mu)$, satisfy non-negativeness, the following equalities (by feasibility)

$$
\text { [1] } u_{12}+v_{2}=4 \quad[2] \quad u_{21}+v_{1}=4
$$

and (by pairwise-stability) inequalities

$$
\text { [3] } u_{12}+v_{1} \geq 5 \quad[4] \quad u_{21}+v_{2} \geq 2,
$$

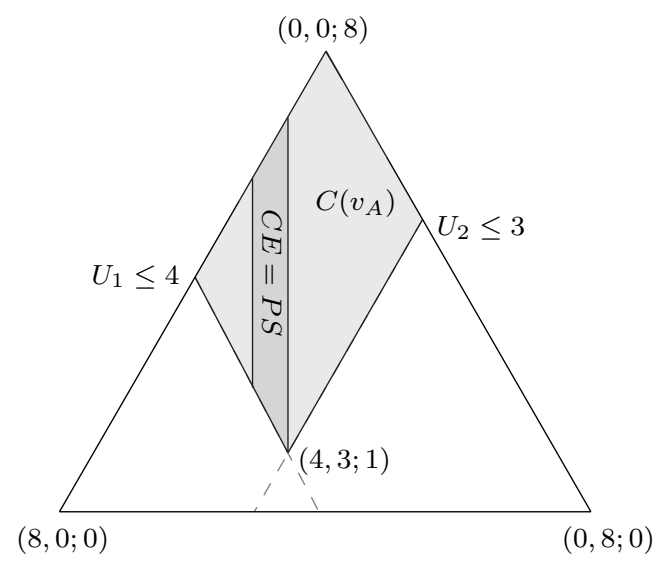

Figure 1: $C\left(v_{A}\right) \nsupseteq C E(A)=P S(A)$

Making use of [3] and [2], we get $u_{12}-u_{21} \geq 1$. Moreover, from [4] and [1], we get $u_{21}-u_{12} \geq-2$. Then, $U_{1}-U_{2} \geq 1$ and $U_{2}-U_{1} \geq-2$, where the payoff for buyer 1 is $U_{1}=u_{12}$ because of his unitary capacity, and similarly for buyer 2.

If $C E(A)$ denotes the set of competitive equilibria payoff vectors and $P S(A)$ denotes the set of payoff vectors associated to the pairwise-stable outcomes, then we get $C\left(v_{A}\right) \nsupseteq$ $C E(A)=P S(A)$. In Figure 1, we depict them as a subset of the core of the game.

Notice that in this case, the buyers-optimal core allocation $(4,3 ; 1)$ does come from a competitive equilibrium while the seller optimal does not. It is easy to find instances, in which the buyers-optimal core allocation does not come from a competitive equilibrium.

Example 4.6. Consider a market with only one seller and two buyers. The seller owns three objects and each buyer can acquire two objects. For the purposes of this example, we show only one dummy object. Therefore, this market $(M,\{0\}, Q, A, r)$ is given by $M=\{1,2\}, Q=\left\{1^{\prime}, 2^{\prime}, 3^{\prime}, j_{0}\right\}$ and $r=(2,2)$. The valuation matrix $A$ is the following 


$$
\begin{aligned}
& 1^{\prime} \\
& 2
\end{aligned}\left(\begin{array}{cccc}
8 & 2^{\prime} & 3^{\prime} & j_{0} \\
10 & 3 & 6 & 0 \\
10 & 1 & 2 & 0
\end{array}\right)
$$

where the unique optimal matching is circled. Consider the related one-seller assignment game $\left(M \cup\{0\}, v_{A}\right)$. The buyers-optimal core allocation is $(7,5 ; 7)$. Assume that $(p, \mu)$ is a competitive equilibrium such that $(U(p, \mu) ; V(p, \mu))=(7,5 ; 7)$. Notice that there is a unique optimal matching $\mu \in \mathcal{M}_{A}(M, Q)$. Therefore, we have that $U_{1}(p, \mu)=$ $\sum_{j \in \mu(1)}\left(a_{1 j}-p_{j}\right)=7$, and then $p_{2}+p_{3}=2$. Notice that $a_{22}+a_{23}-p_{2}-p_{3}=1$ and since $2 \geq p_{j} \geq 0$ for $j \in\left\{2^{\prime}, 3^{\prime}\right\}$, then $a_{2 j}-p_{j}>0$ for some $j \in\left\{2^{\prime}, 3^{\prime}\right\}$. This contradicts that $(p, \mu)$ is a competitive equilibrium because $j_{0} \in \mu(2)$ and $a_{20}-p_{0}=0$.

Since the set of competitive equilibria payoff vectors may be strictly included in the core, we want to analyze when these two sets coincide. As a first step to study under which conditions all core allocations are competitive, we characterize the fact that the seller-optimal core allocation is a payoff vector associated to some competitive equilibrium. To this end, let us first define the set of desirable objects, $Q_{A}^{*}$. We say that an object is desirable if at least one buyer valuates it positively

$$
Q_{A}^{*}=\left\{j \in Q \mid a_{i j}>0 \text { for some } i \in M\right\} .
$$

The conditions for the one-seller optimal core allocation to be a competitive equilibrium payoff vector require that each object sold is acquired by the buyer who valuates it the most and that all desirable objects are sold.

Lemma 4.7. Let $(M,\{0\}, Q, A, r)$ be a one-seller assignment market with multi-unit demands. The seller-optimal core allocation is a competitive equilibrium payoff vector if and only if there is an optimal matching $\mu \in \mathcal{M}_{A}(M, Q)$ and the following two conditions are satisfied:

(a) For all $j \in \mu(M)$ and all $i \in M \backslash\left\{\mu^{-1}(j)\right\}, a_{i j} \leq a_{\mu^{-1}(j) j}$,

(b) $Q_{A}^{*} \subseteq \mu(M)$.

Proof. We first prove the 'if' part. Assume that $\mu \in \mathcal{M}_{A}(M, Q)$ satisfies conditions $(a)$ and $(b)$. Define $p_{j}=a_{\mu^{-1}(j) j}$ for all $j \in \mu(M)$ and $p_{j}=0$ for all $j \in Q \backslash \mu(M)$. We show that $\mu(i) \in D_{i}(p)$ for all $i \in M$. Take any $i \in M$ and consider any $R \in 2_{r_{i}}^{Q}$. Since $\mu$ satisfies $(a)$ and $(b)$, and by definition of $p$,

$$
\sum_{j \in R}\left(a_{i j}-p_{j}\right)=\sum_{j \in R \cap \mu(M)}\left(a_{i j}-a_{\mu^{-1}(j) j}\right)+\sum_{j \in R \backslash \mu(M)}\left(a_{i j}-0\right) \leq 0=\sum_{j \in \mu(i)}\left(a_{i j}-p_{j}\right),
$$

and thus $\mu(i) \in D_{i}(p)$ for all $i \in M$. Besides, by definition of $p$, we get $p_{j}=0$ for each $j \in Q \backslash \mu(M)$. Notice that $(U(p, \mu), V(p, \mu))$ is the seller-optimal core allocation.

Now, we prove the 'only if' part. Assume that $(p, \mu)$ is a competitive equilibrium and $(U(p, \mu), V(p, \mu))$ is the seller-optimal core allocation. By $\mathrm{R} 1$ in page 7 , we have that $\mu \in \mathcal{M}_{A}(M, Q)$. Moreover, in the seller-optimal core allocation the seller's payoff is equal to $v_{A}(M \cup\{0\})$.

We claim that

$$
p_{j}=a_{\mu^{-1}(j) j} \text { for all } j \in \mu(M) \text {. }
$$


If $p_{j}>a_{\mu^{-1}(j) j}$ for some $j \in \mu(M)$, then for all $R \in D_{\mu^{-1}(j)}(p)$ we have $j \notin R$, and as a consequence $(p, \mu)$ is not a competitive equilibrium. On the other hand, if $p_{j}<a_{\mu^{-1}(j) j}$ for some $j \in \mu(M)$ then $\sum_{j \in Q} p_{j}<v_{A}(M \cup\{0\})$ and the seller-optimal core allocation is not the payoff vector of $(p, \mu)$. Taking (13) into account, we shall prove that $\mu$ satisfies (a). Assume on the contrary that there is some $i \in M$ such that $a_{i j}>a_{\mu^{-1}(j) j}$ for some $j \in Q$ with $i \in M \backslash\left\{\mu^{-1}(j)\right\}$. Let $R \in 2_{r_{i}}^{Q}$ be the package formed by object $j$ and copies of the dummy object, i.e., $R=\left\{j, j_{0}^{1}, j_{0}^{2}, \ldots, j_{0}^{r_{i}-1}\right\}$. Since $\sum_{j \in R}\left(a_{i j}-p_{j}\right)>$ $0=\sum_{j \in \mu(i)}\left(a_{i j}-p_{j}\right)$, we obtain that $\mu(i) \notin D_{i}(p)$ in contradiction with $(p, \mu)$ being a competitive equilibrium. Then $\mu$ satisfies $(a)$. In order to show $(b)$, assume on the contrary that, there is some $j \in Q_{A}^{*} \backslash \mu(M)$. By definition of competitive equilibrium, the price of this object is $p_{j}=0$. Since $j \in Q_{A}^{*}$, there is some $i \in M$ such that $a_{i j}>0$. This implies that $\mu(i) \notin D_{i}(p)$ because $\sum_{j \in R}\left(a_{i j}-p_{j}\right)>\sum_{j \in \mu(i)}\left(a_{i j}-p_{j}\right)$ where $R=\left\{j, j_{0}^{1}, j_{0}^{2}, \ldots, j_{0}^{r_{i}-1}\right\}$ and $R \in 2_{r_{i}}^{Q}$. This contradicts $(p, \mu)$ being a competitive equilibrium. Hence, $\mu$ satisfies $(b)$.

Notice that Condition $(a)$ on Lemma 4.7 is not satisfied in Example 4.5, while in Example 4.6 is satisfied.

The following theorem is the main result of this section. We characterize the coincidence between the set of competitive equilibria payoff vectors and the core of the one-seller assignment game.

Theorem 4.8. Let $(M,\{0\}, Q, A, r)$ be the one-seller assignment market with multi-unit demands and $\left(M \cup\{0\}, v_{A}\right)$ be its associated one-seller assignment game. Then the core of $\left(M \cup\{0\}, v_{A}\right)$ coincides with the set of competitive equilibria payoff vectors if and only if there is an optimal matching $\mu \in \mathcal{M}_{A}(M, Q)$ which satisfies the following three conditions:

(a) For all $j \in \mu(M)$ and all $i \in M \backslash\left\{\mu^{-1}(j)\right\}, a_{i j} \leq a_{\mu^{-1}(j) j}$,

(b) $Q_{A}^{*} \subseteq \mu(M)$,

(c) $M_{i}^{v_{A}} \leq \sum_{j \in \mu(i)}\left(a_{i j}-\max _{t \in M \backslash\{i\}}\left\{a_{t j}\right\}\right)$ for all $i \in M$.

Proof. We first prove the 'if' part. Assume that some $\mu \in \mathcal{M}_{A}(M, Q)$ satisfies $(a)$, $(b)$ and $(c)$. We show that any $(U, V) \in C\left(v_{A}\right)$ is the payoff vector of some competitive equilibrium. By conditions $(a)$ and $(c)$, for each $i \in M$, we can find some $\left(\alpha_{i j}\right)_{j \in \mu(i)} \in \mathbb{R}^{r_{i}}$ such that $a_{i j} \geq \alpha_{i j} \geq \max _{t \in M \backslash\{i\}}\left\{a_{t j}\right\}$ for all $j \in \mu(i)$ and $M_{i}^{v_{A}}=\sum_{j \in \mu(i)}\left(a_{i j}-\alpha_{i j}\right)$. Take any $(U, V) \in C\left(v_{A}\right)$ and define $b_{i}=M_{i}^{v_{A}}-U_{i}$ for all $i \in M$. Since for all $i \in M$ we have $M_{i}^{v_{A}} \geq U_{i} \geq 0$ then $M_{i}^{v_{A}} \geq b_{i} \geq 0$.

Let us define $p \in \mathbb{R}^{Q}$ by

$$
p_{j}= \begin{cases}\alpha_{\mu^{-1}(j) j}+\frac{a_{\mu^{-1}(j) j}-\alpha_{\mu^{-1}(j) j}}{M_{\mu^{-1}(j)}} b_{\mu^{-1}(j)} & \text { for all } j \in \mu(M) \text { and } M_{\mu^{-1}(j)}^{v_{A}}>0, \\ a_{\mu^{-1}(j) j} & \text { for all } j \in \mu(M) \text { and } M_{\mu^{-1}(j)}^{v_{A}}=0, \\ 0 & \text { for all } j \in Q \backslash \mu(M) .\end{cases}
$$

Notice that $p$ is a feasible price vector. Therefore, we show that $\mu(i) \in D_{i}(p)$ for all $i \in M$. It is sufficient to see that $a_{i j}-p_{j} \geq a_{i k}-p_{k}$ for all $j \in \mu(i)$ and all $k \in Q \backslash \mu(i)$. To this end, let us see that for all $i \in M$ and all $j \in \mu(i)$ it holds $a_{i j}-p_{j} \geq 0$ while 
$a_{i k}-p_{k} \leq 0$ for all $k \in Q \backslash \mu(i)$. On one hand, take $i \in M$ such that $M_{i}^{v_{A}}>0$. Then $a_{i j}-p_{j}=a_{i j}-\alpha_{i j}-\frac{a_{i j}-\alpha_{i j}}{M_{i}^{v_{A}}} b_{i}=\left(a_{i j}-\alpha_{i j}\right)\left(1-\frac{b_{i}}{M_{i}^{v^{\prime} A}}\right) \geq 0$ for all $j \in \mu(i)$. Now, take $i \in M$ such that $M_{i}^{v_{A}}=0$. Then $a_{i j}-p_{j}=a_{i j}-a_{i j}=0$ for all $j \in \mu(i)$. On the other hand, take $k \in \mu(M)$ such that $M_{\mu^{-1}(k) k}^{v_{A}}>0$. Then for any $i \in M \backslash\left\{\mu^{-1}(k)\right\}$, we have

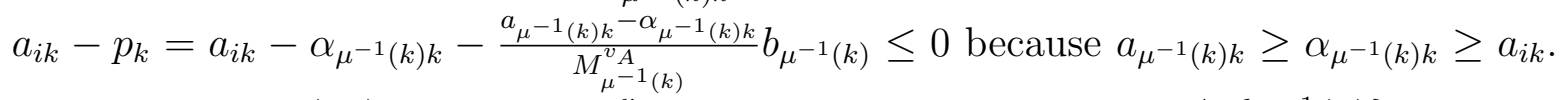
Now, take $k \in \mu(M)$ such that $M_{\mu^{-1}(k) k}^{v_{A}}=0$. Then for any $i \in M \backslash\left\{\mu^{-1}(k)\right\}$, we have $a_{i k}-p_{k}=a_{i k}-a_{\mu^{-1}(k) k} \leq 0$ because of assumption $(a)$. Finally, consider $k \in Q \backslash \mu(M)$. Then for any $i \in M, a_{i k}-p_{k}=0$ because of $(b)$. Thus $\mu(i) \in D_{i}(p)$ for all $i \in M$. Moreover, by definition of $p, p_{j}=0$ for all $j \in Q \backslash \mu(M)$. Hence, $(p, \mu)$ is a competitive equilibrium. Then, the payoffs are

$$
\begin{aligned}
U_{i}(p, \mu) & =\sum_{j \in \mu(i)}\left(a_{i j}-p_{j}\right)=\sum_{j \in \mu(i)}\left(a_{i j}-\alpha_{i j}-\frac{a_{i j}-\alpha_{i j}}{M_{i}^{v_{A}}} b_{i}\right) \\
& =\sum_{j \in \mu(i)}\left(a_{i j}-\alpha_{i j}\right)\left(1-\frac{b_{i}}{M_{i}^{v_{A}}}\right)=M_{i}^{v_{A}}-b_{i}=U_{i}
\end{aligned}
$$

for all $i \in M$ such that $M_{i}^{v_{A}}>0$, where the last equality comes from $\sum_{j \in \mu(i)}\left(a_{i j}-\alpha_{i j}\right)=$ $M_{i}^{v_{A}}$. Take now any $i \in M$ such that $M_{i}^{v_{A}}=0$. From the definition of $p_{j}$ in (14), we have

$$
U_{i}(p, \mu)=\sum_{j \in \mu(i)}\left(a_{i j}-p_{j}\right)=\sum_{j \in \mu(i)}\left(a_{i j}-a_{i j}\right)=0=U_{i}
$$

since $(U(p, \mu), V(p, \mu)) \in C\left(v_{A}\right)$ for any competitive equilibrium $(p, \mu)$, by efficiency the seller's payoff is $V(p, \mu)=v_{A}(M \cup\{0\})-\sum_{i \in M} U_{i}(p, \mu)=v_{A}(M \cup\{0\})-\sum_{i \in M} U_{i}=V$. This completes the proof of the "if" part.

Now, we prove the 'only if' part. Assume that the core and the set of payoff vectors associated to the competitive equilibria coincide. By Lemma 4.7, conditions $(a)$ and (b) hold for some optimal matching. Then, we only have to prove $(c)$. Assume on the contrary that for each $\mu \in \mathcal{M}_{A}(M, Q)$, there is some buyer $i^{\prime} \in M$ such that $M_{i^{\prime}}^{v_{A}}>\sum_{j \in \mu\left(i^{\prime}\right)}\left(a_{i^{\prime} j}-\max _{t \in M \backslash\left\{i^{\prime}\right\}}\left\{a_{t j}\right\}\right)$. Recall the description of the core in (5) and consider $(U, V) \in C\left(v_{A}\right)$ with $U_{i^{\prime}}=M_{i^{\prime}}^{v_{A}}$ for the buyer $i^{\prime}$ and $U_{i}=0$ for all $i \in$ $M \backslash\left\{i^{\prime}\right\}$. By assumption, there is a competitive equilibrium $(p, \mu)$ such that $(U, V)$ is its payoff vector. Take this competitive equilibrium price vector $p$ and the matching $\mu \in \mathcal{M}_{A}(M, Q)$ such that $M_{i^{\prime}}^{v_{A}}>\sum_{j \in \mu\left(i^{\prime}\right)}\left(a_{i^{\prime} j}-\max _{t \in M \backslash\left\{i^{\prime}\right\}}\left\{a_{t j}\right\}\right)$. Then $(p, \mu)$ is a competitive equilibrium (recall R1 in page 7). Therefore $p_{j}=a_{\mu^{-1}(j) j}$ for all $j \in$ $\mu\left(M \backslash\left\{i^{\prime}\right\}\right)$ and $\sum_{j \in \mu\left(i^{\prime}\right)} p_{j}=\sum_{j \in \mu\left(i^{\prime}\right)} a_{i^{\prime} j}-M_{i^{\prime}}^{v_{A}}$. We obtain $\sum_{j \in \mu\left(i^{\prime}\right)} a_{i^{\prime} j}-\sum_{j \in \mu\left(i^{\prime}\right)} p_{j}=$ $M_{i^{\prime}}^{v_{A}}>\sum_{j \in \mu\left(i^{\prime}\right)}\left(a_{i^{\prime} j}-\max _{i \in M \backslash\left\{i^{\prime}\right\}}\left\{a_{i j}\right\}\right)$. As a consequence, $\sum_{j \in \mu\left(i^{\prime}\right)} \max _{i \in M \backslash\left\{i^{\prime}\right\}}\left\{a_{i j}\right\}>$ $\sum_{j \in \mu\left(i^{\prime}\right)} p_{j}$ which implies that there is some $i \in M \backslash\left\{i^{\prime}\right\}$ such that $a_{i j}>p_{j}$ for some $j \in \mu\left(i^{\prime}\right)$. We have that $\mu(i) \notin D_{i}(p)$ because $a_{i k}-p_{k}=0<a_{i j}-p_{j}$ for all $k \in \mu(i)$ and $j \notin \mu(i)$. This contradicts that $(p, \mu)$ is a competitive equilibrium. Hence condition $(c)$ holds.

Notice that as a consequence of the proof of Theorem 4.8, if an assignment market satisfies $(a),(b)$ and $(c)$ for some $\mu \in \mathcal{M}_{A}(M, Q)$, it also does for any $\mu^{\prime} \in \mathcal{M}_{A}(M, Q)$. 
Our next example shows that when a core element is a competitive equilibrium payoff vector, then the associated equilibrium prices may be not unique.

Example 4.9. Consider a market with only one seller and two buyers. The seller owns four objects on sale. Each buyer can acquire two objects. For the purposes of this example, we do not show dummy objects. That is, the market $(M,\{0\}, Q, A, r)$ is given by $M=\{1,2\}, Q=\left\{1^{\prime}, 2^{\prime}, 3^{\prime}, 4^{\prime}\right\}$ and $r=(2,2)$. The valuation matrix $A$ is the following

$$
\begin{gathered}
1 \\
2\left(\begin{array}{cccc}
1^{\prime} & 2^{\prime} & 3^{\prime} & 4^{\prime} \\
0 & 2 & 0 & 0 \\
0 & 3 & 3
\end{array}\right),
\end{gathered}
$$

where the unique optimal matching is circled. Consider the game $\left(M \cup\{0\}, v_{A}\right)$ and notice that $M_{1}^{v_{A}}=4$ and $M_{2}^{v_{A}}=6$. Take $(U, V)=(3,4 ; 3) \in C\left(v_{A}\right)$. Since conditions $(a),(b)$ and $(c)$ of Theorem 4.8 are satisfied, we can define a competitive equilibrium $(p, \mu)$ associated to $(U, V)$ following the proof of the theorem. Since $M_{i}^{v_{A}}=\sum_{j \in \mu(i)} a_{i j}$ for all $i \in M, b_{1}=M_{1}^{v_{A}}-U_{1}=1$ and $b_{2}=M_{2}^{v_{A}}-U_{2}=2$, let $p_{j}=\frac{a_{1 j}}{\sum_{j \in \mu(1)} a_{1 j}} \cdot 1=0.5$ for $j=1^{\prime}, 2^{\prime}$ and $p_{j}=\frac{a_{2 j}}{\sum_{j \in \mu(2)} a_{2 j}} \cdot 2=1$ for $j=3^{\prime}, 4^{\prime}$. Therefore with $p=(0.5,0.5,1,1)$ the pair $(p, \mu)$ is a competitive equilibrium and its associated payoff vector is $(U, V)$. Take now $p^{\prime}=(1,0,1.5,0.5)$ and notice that $\left(p^{\prime}, \mu\right)$ is also a competitive equilibrium and $\left(U\left(p^{\prime}, \mu\right), V\left(p^{\prime}, \mu\right)\right)=(3,4 ; 3)$. Besides, take $p^{\prime \prime}=(0,1,0.5,1.5)$ and $\left(p^{\prime \prime}, \mu\right)$ is also a competitive equilibrium with $\left(U\left(p^{\prime \prime}, \mu\right), V\left(p^{\prime}, \mu\right)\right)=(3,4 ; 3)$.

Notwithstanding, if the buyers-optimal core allocation comes from a competitive equilibrium then the prices are uniquely determined. Assume on the contrary that there exist two competitive equilibrium prices $p$ and $p^{\prime}\left(p \neq p^{\prime}\right)$ such that their associated payoff vector is the buyers-optimal core allocation. Since $p \neq p^{\prime}$, let us assume w.l.o.g. that there is some $j \in Q$ such that $p_{j}>p_{j}^{\prime}$. This implies that, for any $\mu \in \mathcal{M}_{A}(M, Q)$, object $j \in \mu\left(i^{\prime}\right)$ for some $i^{\prime} \in M$, because $p_{j}>p_{j}^{\prime} \geq 0$. Since the set of competitive equilibrium prices has the structure of a complete lattice, then $p^{\prime \prime}=p \wedge p^{\prime}$ is also a competitive equilibrium price. Notice that $M_{i^{\prime}}^{v_{A}}=\sum_{j \in \mu\left(i^{\prime}\right)}\left(a_{i^{\prime} j}-p_{j}\right)<\sum_{j \in \mu\left(i^{\prime}\right)}\left(a_{i^{\prime} j}-p_{j}^{\prime \prime}\right)$ which contradicts that $p^{\prime \prime}$ is a competitive equilibrium price because the payoff vector associated to $\left(p^{\prime \prime}, \mu\right)$ does not belong to the core.

Nevertheless, convexity is a sufficient condition for the buyers-optimal core allocation to come from a competitive equilibrium. To see that, take a one-seller assignment market with multi-unit demands $(M,\{0\}, Q, A, r)$, such that the related one-seller assignment game $\left(M \cup\{0\}, v_{A}\right)$ is convex. By Proposition 3.2, for any $\mu \in \mathcal{M}_{A}(M, Q)$ we have

$$
\sum_{j \in \mu(i)} a_{i j} \geq \sum_{j \in R} a_{i j}
$$

for all $R \in 2_{r_{i}}^{Q}$ and all $i \in M$. It is straightforward to see that $(p, \mu)$ is a competitive equilibrium, where $p=(0, \ldots, 0) \in \mathbb{R}_{+}^{Q}$. Indeed, the payoff vector associated to $(p, \mu)$ is the buyers-optimal core allocation. 


\section{A buying and selling procedure for the buyers- optimal core allocation}

When agents in one-seller assignment markets behave strategically, we analyze a procedure that attains the best core payoff for all buyers. Under the assumption of complete information, we give the following two-phase procedure $\Gamma$. In the first phase, all buyers play. Each buyer announces a tentative package of objects that he would accept to buy at some price. All these requests are simultaneous. In the second phase, the seller, after observing all these requests, chooses a set of buyers and allocates to each of them a package at a price that makes the buyer indifferent with his initial request.

In more detail, the two phases of the procedure $\Gamma$ are:

1. Buyers choose strategies simultaneously. A pure strategy for a buyer $i \in M$, denoted by $s_{i}$, consists of a pair formed by a package and its price, $\left(R(i), P_{R(i)}\right) \in$ $2_{r_{i}}^{Q} \times \mathbb{R}_{+}$. Denote by $s=\left(s_{1}, \ldots, s_{m}\right)$ a buyers' strategy profile.

2. Given a buyers' strategy profile $s$ and making use of the complete information assumption, the seller chooses: a) a coalition of buyers $S^{*} \subseteq M$; b) a matching $\mu \in \mathcal{M}\left(S^{*}, Q\right)$; and c) a price $P_{\mu(i)}^{*} \in \mathbb{R}_{+}$for each $i \in S^{*}$ such that

$$
\sum_{j \in \mu(i)} a_{i j}-P_{\mu(i)}^{*} \geq \sum_{j \in R(i)} a_{i j}-P_{R(i)}
$$

where $\left(R(i), P_{R(i)}\right)=s_{i}$. We denote by $\mathcal{O}(s)$ the seller's strategy.

If a buyer $i \in S^{*}$ is matched by the selected matching $\mu \in \mathcal{M}\left(S^{*}, Q\right)$, he receives the package $\mu(i)$ and pays the price $P_{\mu(i)}^{*}$. If a buyer does not receive a package, he pays nothing. Therefore the final outcome is $\Gamma(s, \mathcal{O}(s))=\left(\mu, P^{*}\right)$ where $\mu \in \mathcal{M}\left(S^{*}, Q\right)$ and $P^{*}=\left(P_{\mu(i)}^{*}\right)_{i \in S^{*}}$. The payoffs are given by

$$
\begin{array}{ll}
U_{i}\left(\mu, P^{*}\right)= \begin{cases}\sum_{j \in \mu(i)} a_{i j}-P_{\mu(i)}^{*}, & \text { if } i \in S^{*} \\
0 & \text { if } i \in M \backslash S^{*}\end{cases} \\
V\left(\mu, P^{*}\right)=\sum_{i \in S^{*}} P_{\mu(i)}^{*} & \text { for the seller. }
\end{array}
$$

Now, we introduce some notation. Given a buyers' strategy profile $s$ and $T \subseteq S$, we say that a matching $\mu \in \mathcal{M}(S, Q)$ is a matching compatible with $s_{-(S \backslash T)}$ if $\sum_{j \in \mu(i)} a_{i j} \geq$ $\sum_{j \in R(i)} a_{i j}-P_{R(i)}$ for each $i \in T$ where $\left(R(i), P_{R(i)}\right)=s_{i}$. We write $\mu \in \mathcal{M}^{s_{-}(S \backslash T)}(S, Q)$. When $S \backslash T=\{i\}$, we simply write $\mathcal{M}^{s_{-i}}(S, Q)$. Moreover, we define the set $\mathcal{P}(\mu, T)$ of all price vectors $P \in \mathbb{R}_{+}^{T}$ for packages assigned to agents in $T$, that satisfies (15) for each $i \in T$. Notice that if a matching $\mu \in \mathcal{M}(S, Q)$ is compatible with $s_{-(S \backslash T)}$, this is equivalent to saying that $\mathcal{P}(\mu, T) \neq \emptyset$. We identify with $\mathcal{M}_{A}^{s_{-}(S \backslash T)}(S, Q)$ the set of optimal matchings for $\left(S,\{0\}, Q, A_{\mid S \times Q}, r_{\mid S}\right)$ compatible with $s_{-(S \backslash T)}$. Therefore, notice that the seller chooses only matchings that are compatible with the buyers' requests.

Our next proposition characterizes the Subgame Perfect Equilibrium (SPE) of the procedure $\Gamma$ by means of three conditions. 
Proposition 5.1. Let $(M,\{0\}, Q, A, r)$ be a one-seller assignment market with multiunit demands and $(s, \mathcal{O}(s))$ be a strategy profile for $\Gamma$. Then $(s, \mathcal{O}(s))$ is a SPE of $\Gamma$ if and only if the following conditions hold:

(a) The seller chooses $S^{*} \subseteq M, \mu \in \mathcal{M}^{s}\left(S^{*}, Q\right)$ and $P^{*} \in \mathcal{P}\left(\mu, S^{*}\right)$ such that for all $S \subseteq M, \mu^{\prime} \in \mathcal{M}^{s}(S, Q)$ and $P \in \mathcal{P}\left(\mu^{\prime}, S\right)$, it holds

$$
\sum_{i \in S^{*}} P_{\mu(i)}^{*} \geq \sum_{i \in S} P_{\mu^{\prime}(i)}
$$

(b) For all $i^{\prime} \in S^{*}$, there exist $S \subseteq M \backslash\left\{i^{\prime}\right\}, \mu^{\prime} \in \mathcal{M}^{s}(S, Q)$ and $P \in \mathcal{P}\left(\mu^{\prime}, S\right)$ such that

$$
\sum_{i \in S^{*}} P_{\mu(i)}^{*}=\sum_{i \in S} P_{\mu^{\prime}(i)}
$$

(c) For all $t \in M \backslash S^{*}$, all $S \subseteq M \backslash\{t\}$, all $\mu^{\prime} \in \mathcal{M}^{s_{-t}}(S \cup\{t\}, Q)$ and all $P \in \mathcal{P}\left(\mu^{\prime}, S\right)$

$$
\sum_{i \in S^{*}} P_{\mu(i)}^{*} \geq \sum_{i \in S} P_{\mu^{\prime}(i)}+\sum_{j \in \mu^{\prime}(t)} a_{t j}
$$

Proof. We prove the "if" part. We see that under conditions $(a),(b)$ and $(c),(s, \mathcal{O}(s))$ is a SPE.

Condition $(a)$ guarantees that the seller is playing a best response to the buyers' strategy profile, since the selected matching and the price vector maximize the seller's payoff. Notice that condition (a) implies that the seller chooses $\mu \in \mathcal{M}^{s}\left(S^{*}, Q\right)$ and $P^{*} \in \mathcal{P}\left(\mu, S^{*}\right)$ such that $P_{\mu(i)}^{*}$ satisfies (15) as an equality for each $i \in S^{*}$.

Condition $(b)$ guarantees that buyer $i^{\prime} \in S^{*}$ is playing a best response to the other agents' strategy profile. On one hand, assume that buyer $i^{\prime} \in S^{*}$ unilaterally modifies his request to $\left(R^{\prime}\left(i^{\prime}\right), P_{R^{\prime}\left(i^{\prime}\right)}^{\prime}\right)$ such that $\sum_{j \in R^{\prime}\left(i^{\prime}\right)} a_{i^{\prime} j}-P_{R^{\prime}\left(i^{\prime}\right)}^{\prime}>\sum_{j \in R\left(i^{\prime}\right)} a_{i^{\prime} j}-P_{R\left(i^{\prime}\right)}$. This means that all his current acceptable packages require a lower price. Because of $(b)$, the seller will maximize her payoff at the matching $\mu^{\prime}$ leaving him unassigned. Therefore buyer $i^{\prime}$ will not be better off. On the other hand, if buyer $i^{\prime} \in S^{*}$ requests $\left(R^{\prime}\left(i^{\prime}\right), P_{R^{\prime}\left(i^{\prime}\right)}^{\prime}\right)$ such that $\sum_{j \in R^{\prime}\left(i^{\prime}\right)} a_{i^{\prime} j}-P_{R^{\prime}\left(i^{\prime}\right)}^{\prime}<\sum_{j \in R\left(i^{\prime}\right)} a_{i^{\prime} j}-P_{R\left(i^{\prime}\right)}$, then he will acquire some package but at a price that makes him worse off.

Condition $(c)$ guarantees that each buyer $t \in M \backslash S^{*}$ is also playing a best response. Indeed, this condition ensures that buyer $t \in M \backslash S^{*}$ has no chance to unilaterally changing his request $\left(R^{\prime}(t), P_{R^{\prime}(t)}^{\prime}\right)$ and being matched by the seller. This is because if his request satisfies $\sum_{j \in R^{\prime}(t)} a_{t j}-P_{R^{\prime}(t)}^{\prime}=0$, because of $(c)$, the seller will choose the same matching, and buyer $t$ will remain unassigned. On the other hand, it is obvious that if buyer $t$ requests $\left(R^{\prime}(t), P_{R^{\prime}(t)}^{\prime}\right)$ such that $\sum_{j \in R^{\prime}(t)} a_{t j}-P_{R^{\prime}(t)}^{\prime}>\sum_{j \in R(t)} a_{t j}-P_{R(t)}$, he will also remain unassigned. Then $(s, \mathcal{O}(s))$ is a SPE.

Let us prove the "only if" part. Condition $(a)$ is satisfied in any SPE since it states that, given any buyers' strategy profile $s$, the seller maximizes her payoff.

To prove $(b)$, assume on the contrary that $(s, \mathcal{O}(s))$ is a SPE and there exists $i^{\prime} \in S^{*}$, for all $S \subseteq M \backslash\left\{i^{\prime}\right\}$, all $\mu^{\prime} \in \mathcal{M}^{s}(S, Q)$ and all $P^{\prime} \in \mathcal{P}\left(\mu^{\prime}, S\right)$

$$
\sum_{i \in S^{*}} P_{\mu(i)}^{*}>\sum_{i \in S} P_{\mu^{\prime}(i)}^{\prime}
$$


Notice that $P_{\mu\left(i^{\prime}\right)}^{*}>0$, since otherwise $S^{*} \backslash\left\{i^{\prime}\right\}$ would satisfy condition (b) in contradiction with our assumption. Therefore, buyer $i^{\prime}$ has incentives to deviate by slightly decreasing the price of his request in such a way that inequality (18) is still maintained. This contradicts that $(s, \mathcal{O}(s))$ is a SPE. Hence condition $(b)$ must hold.

In order to prove $(c)$, assume on the contrary that for some $t \in M \backslash S^{*}$, there exists $S \subseteq M \backslash\{t\}$, some $\mu^{\prime} \in \mathcal{M}^{s_{-t}}(S \cup\{t\}, Q)$ and $P^{\prime} \in \mathcal{P}\left(\mu^{\prime}, S\right)$ such that

$$
\sum_{i \in S^{*}} P_{\mu(i)}^{*}<\sum_{i \in S} P_{\mu^{\prime}(i)}^{\prime}+\sum_{j \in \mu^{\prime}(t)} a_{t j}
$$

Notice that $\sum_{j \in \mu^{\prime}(t)} a_{t j}>0$, since otherwise matching $\mu^{\prime \prime}=\left\{(i, j) \in \mu^{\prime} \mid i \in S\right\}$ satisfies $\mu^{\prime \prime} \in \mathcal{M}^{s}(S, Q)$ and

$$
\sum_{i \in S^{*}} P_{\mu(i)}^{*}<\sum_{i \in S} P_{\mu^{\prime \prime}(i)}^{\prime}
$$

which contradicts (16) and the fact that $(s, \mathcal{O}(s))$ is a SPE. Since $\sum_{j \in \mu^{\prime}(t)} a_{t j}>0$, buyer $t$ has incentives to deviate setting $\left(\mu^{\prime}(t), P_{\mu^{\prime}(t)}\right)$ such that $\sum_{j \in \mu^{\prime}(t)} a_{t j}-P_{\mu^{\prime}(t)}>0$ and

$$
\sum_{i \in S} P_{\mu^{\prime}(i)}^{\prime}+P_{\mu^{\prime}(t)}>\sum_{i \in S^{*}} P_{\mu(i)}^{*},
$$

in order to receive $\mu^{\prime}(t)$ with a positive payoff. This contradicts that $(s, \mathcal{O}(s))$ is a SPE. Hence condition $(c)$ of Proposition 5.1 must hold and this concludes the proof.

Now, let us introduce the following notation. Given a buyers' strategy profile $s$, a matching $\mu \in \mathcal{M}(S, Q)$ and $T \subseteq S$, we denote by $\bar{P} \in \mathcal{P}(\mu, T)$ the price vector such that

$$
\sum_{j \in \mu(i)} a_{i j}-\bar{P}_{\mu(i)}=\sum_{j \in R(i)} a_{i j}-P_{R(i)} \text { for all } i \in T
$$

where $\left(R(i), P_{R(i)}\right)=s_{i}$.

Our following result describes a SPE of $\Gamma$ in which the payoff vector is the buyersoptimal core allocation of the one-seller assignment game.

Theorem 5.2. Let $(M,\{0\}, Q, A, r)$ be a one-seller assignment market with multi-unit demands and $\left(M \cup\{0\}, v_{A}\right)$ be its one-seller assignment game. Then the buyers-optimal core allocation of $\left(M \cup\{0\}, v_{A}\right)$ is attained in a SPE of $\Gamma$.

Proof. Let $s$ be the following buyers' strategy profile: each buyer $i \in M$ announces $\left(R(i), P_{R(i)}\right)$ such that $\sum_{j \in R(i)} a_{i j}-P_{R(i)}=M_{i}^{v_{A}}$. The seller chooses $S^{*}=M$, any $\mu \in \mathcal{M}_{A}(M, Q)$ and $P^{*}=\bar{P} \in \mathcal{P}(\mu, M)$ where $\bar{P} \in \mathcal{P}(\mu, M)$ is as in (19). We prove, by means of Proposition 5.1, that these strategies are a SPE. That proved, it is obvious that the payoff vector will be the buyers-optimal core allocation. First, we show that any $\mu \in \mathcal{M}_{A}(M, Q)$ is compatible with $s$. For any $i^{\prime} \in M$, let $\mu^{\prime} \in \mathcal{M}_{A}\left(M \backslash\left\{i^{\prime}\right\}, Q\right)$, then

$$
\begin{aligned}
M_{i^{\prime}}^{v_{A}} & =v_{A}(M \cup\{0\})-v_{A}\left(\left(M \backslash\left\{i^{\prime}\right\}\right) \cup\{0\}\right)=\sum_{(i, j) \in \mu} a_{i j}-\sum_{(i, j) \in \mu^{\prime}} a_{i j} \\
& \leq \sum_{(i, j) \in \mu} a_{i j}-\sum_{i \in M \backslash\left\{i^{\prime}\right\}} \sum_{j \in \mu(i)} a_{i j}=\sum_{j \in \mu\left(i^{\prime}\right)} a_{i^{\prime} j} .
\end{aligned}
$$


This guarantees that, given $s$, the set of prices $\mathcal{P}(\mu, M) \neq \emptyset$ and then $\mu \in \mathcal{M}_{A}^{s}(M, Q)$.

Now, we prove that the seller maximizes her payoff under $\mu \in \mathcal{M}_{A}^{s}(M, Q)$ and the aforementioned $P^{*} \in \mathcal{P}(\mu, M)$. For any $S \subseteq M$, consider any $\mu^{\prime} \in \mathcal{M}^{s}(S, Q)$ and any $P \in \mathcal{P}\left(\mu^{\prime}, S\right)$. Recall that $\left(M \cup\{0\}, v_{A}\right)$ satisfies buyers-submodular condition (3) and since $S^{*}=M$, we have

$$
\begin{aligned}
\sum_{i \in M} P_{\mu(i)}^{*} & =v_{A}(M \cup\{0\})-\sum_{i \in M} M_{i}^{v_{A}} \geq v_{A}(S \cup\{0\})-\sum_{i \in S} M_{i}^{v_{A}} \\
& \geq \sum_{(i, j) \in \mu^{\prime}} a_{i j}-\sum_{i \in S} M_{i}^{v_{A}} \geq \sum_{i \in S} P_{\mu^{\prime}(i)},
\end{aligned}
$$

where the last inequality is due to $P \in \mathcal{P}\left(\mu^{\prime}, S\right)$. Hence, condition (a) of Proposition 5.1 holds.

In order to prove condition (b) of Proposition 5.1, take any $i^{\prime} \in M$ and consider any $\mu^{\prime} \in \mathcal{M}_{A}\left(M \backslash\left\{i^{\prime}\right\}, Q\right)$. We see that $\mu^{\prime}$ is compatible with $s$. For all $i \in M \backslash\left\{i^{\prime}\right\}$, we have

$$
M_{i}^{v_{A}} \leq v_{A}\left(\left(M \backslash\left\{i^{\prime}\right\}\right) \cup\{0\}\right)-v_{A}\left(\left(M \backslash\left\{i^{\prime}, i\right\}\right) \cup\{0\}\right) \leq \sum_{j \in \mu^{\prime}(i)} a_{i j}
$$

where the first inequality is due to the buyers-submodular condition (2) and the second one because $\sum_{t \in M \backslash\left\{i, i^{\prime}\right\}} \sum_{j \in \mu^{\prime}(t)} a_{t j} \leq v_{A}\left(\left(M \backslash\left\{i, i^{\prime}\right\}\right) \cup\{0\}\right)$. Therefore, $\mathcal{P}\left(\mu^{\prime}, M \backslash\left\{i^{\prime}\right\}\right) \neq$ $\emptyset$ and $\mu^{\prime} \in \mathcal{M}_{A}^{s}\left(M \backslash\left\{i^{\prime}\right\}, Q\right)$. Now, take $i^{\prime} \in M, \mu^{\prime} \in \mathcal{M}_{A}^{s}\left(M \backslash\left\{i^{\prime}\right\}, Q\right)$ and let $\bar{P} \in \mathcal{P}\left(\mu^{\prime}, M \backslash\left\{i^{\prime}\right\}\right)$ be as in (19). Then we have

$$
\begin{aligned}
\sum_{i \in M \backslash\left\{i^{\prime}\right\}} \bar{P}_{\mu^{\prime}(i)} & =v_{A}\left(\left(M \backslash\left\{i^{\prime}\right\}\right) \cup\{0\}\right)-\sum_{i \in M \backslash\left\{i^{\prime}\right\}} M_{i}^{v_{A}} \\
& =v_{A}(M \cup\{0\})-\sum_{i \in M} M_{i}^{v_{A}}=\sum_{i \in M} P_{\mu(i)}^{*} .
\end{aligned}
$$

Therefore, condition (b) of Proposition 5.1 holds.

Condition $(c)$ of Proposition 5.1 is trivially satisfied because $S^{*}=M$. Hence, the described strategies are a SPE.

We have shown that when agents play particular strategies in a SPE of $\Gamma$, the payoff vector is the buyers-optimal core allocation. Now, we prove that this is unique payoff vector of all SPE. First, the next proposition proves that in any SPE of $\Gamma$, the seller selects an optimal matching which gives the worth of the grand coalition.

Proposition 5.3. Let $(M,\{0\}, Q, A, r)$ be a one-seller assignment market with multiunit demands and $(s, \mathcal{O}(s))$ a SPE of $\Gamma$, where $\mathcal{O}(s)$ is formed by the triple $\left(S^{*}, \mu, P^{*}\right)$. Then

$$
\sum_{(i, j) \in \mu} a_{i j}=v_{A}\left(S^{*} \cup\{0\}\right)=v_{A}(M \cup\{0\}) .
$$

Proof. First, we prove $\sum_{(i, j) \in \mu} a_{i j}=v_{A}\left(S^{*} \cup\{0\}\right)$. Assume on the contrary that $(s, \mathcal{O}(s))$ is a $\mathrm{SPE}$ and $\sum_{(i, j) \in \mu} a_{i j}<v_{A}\left(S^{*} \cup\{0\}\right)$. 
Take any $\mu_{1} \in \mathcal{M}_{A}\left(S^{*}, Q\right)$. If $\mu_{1} \in \mathcal{M}_{A}^{s}\left(S^{*}, Q\right)$, take $\bar{P} \in \mathcal{P}\left(\mu_{1}, S^{*}\right)$ as in expression (19). Therefore, by condition (a) of Proposition 5.1, $U_{i}\left(\mu, P^{*}\right)=U_{i}\left(\mu_{1}, \bar{P}\right)$ for all $i \in S^{*}$ and we have

$$
\begin{aligned}
\sum_{i \in S^{*}} \bar{P}_{\mu_{1}(i)} & =\sum_{(i, j) \in \mu_{1}} a_{i j}-\sum_{i \in S^{*}} U_{i}\left(\mu_{1}, \bar{P}\right)=v_{A}\left(S^{*} \cup\{0\}\right)-\sum_{i \in S^{*}} U_{i}\left(\mu_{1}, \bar{P}\right) \\
& >\sum_{(i, j) \in \mu} a_{i j}-\sum_{i \in S^{*}} U_{i}\left(\mu, P^{*}\right)=\sum_{i \in S^{*}} P_{\mu(i)}^{*} .
\end{aligned}
$$

This contradicts (16) and the fact that $(s, \mathcal{O}(s))$ is a SPE. Therefore, $\mu_{1} \notin \mathcal{M}_{A}^{s}\left(S^{*}, Q\right)$.

By applying Lemma A.1, see Appendix A, to $S=T^{\prime}=S^{*}$, there exists $\emptyset \neq T \varsubsetneqq S^{*}$ and $\mu^{\prime} \in \mathcal{M}_{A}^{s}(T, Q)$ such that

$$
\sum_{i \in S^{*} \backslash T}\left(\sum_{j \in R(i)} a_{i j}-P_{R(i)}\right)>v_{A}\left(S^{*} \cup\{0\}\right)-v_{A}(T \cup\{0\}),
$$

where $\left(R(i), P_{R(i)}\right)=s_{i}$ for all $i \in S^{*} \backslash T$.

Consider $\mu^{\prime} \in \mathcal{M}_{A}^{s}(T, Q)$ and take $\bar{P}^{\prime} \in \mathcal{P}\left(\mu^{\prime}, T\right)$ as in expression (19). Therefore, $U_{i}\left(\mu^{\prime}, \bar{P}^{\prime}\right)=U_{i}\left(\mu, P^{*}\right)$ for all $i \in T$. By condition (a) of SPE in Proposition 5.1, we obtain

$$
\begin{aligned}
\sum_{(i, j) \in \mu} a_{i j}-\sum_{i \in S^{*}} U_{i}\left(\mu, P^{*}\right) & =\sum_{i \in S^{*}} P_{\mu(i)}^{*} \geq \sum_{i \in T} \bar{P}_{\mu^{\prime}(i)}^{\prime}=\sum_{(i, j) \in \mu^{\prime}} a_{i j}-\sum_{i \in T} U_{i}\left(\mu^{\prime}, \bar{P}^{\prime}\right) \\
& =v_{A}(T \cup\{0\})-\sum_{i \in T} U_{i}\left(\mu^{\prime}, \bar{P}^{\prime}\right) .
\end{aligned}
$$

Since $T \subseteq S^{*}$, then

$$
\sum_{(i, j) \in \mu} a_{i j}-v_{A}(T \cup\{0\}) \geq \sum_{i \in S^{*} \backslash T} U_{i}\left(\mu, P^{*}\right)=\sum_{i \in S^{*} \backslash T}\left(\sum_{j \in R(i)} a_{i j}-P_{R(i)}\right),
$$

where $\left(R(i), P_{R(i)}\right)=s_{i}$ for all $i \in S^{*} \backslash T$. This contradicts $(20)$. Hence $\sum_{(i, j) \in \mu} a_{i j}=$ $v_{A}\left(S^{*} \cup\{0\}\right)$.

Now, we prove $v_{A}\left(S^{*} \cup\{0\}\right)=v_{A}(M \cup\{0\})$, assume on the contrary that $(s, \mathcal{O}(s))$ is a SPE and $v_{A}\left(S^{*} \cup\{0\}\right)<v_{A}(M \cup\{0\})$. Let $\emptyset \neq T \subseteq M \backslash S^{*}$ be a minimal coalition (with respect to inclusion) in $M \backslash S^{*}$ such that $v_{A}\left(\left(S^{*} \cup T\right) \cup\{0\}\right)>v_{A}\left(S^{*} \cup\{0\}\right)$. Notice that such coalition exists because of monotonicity of $v_{A}$. This implies that there is some $t \in T$ such that $v_{A}\left(\left(S^{*} \cup T\right) \cup\{0\}\right)>v_{A}\left(\left(S^{*} \cup(T \backslash\{t\})\right) \cup\{0\}\right)$. By buyers-submodular condition (2), we have that for all $S \subseteq S^{*} \cup T$ with $t \in S$,

$$
\begin{aligned}
v_{A}(S \cup\{0\}) & -v_{A}((S \backslash\{t\}) \cup\{0\}) \\
& \geq v_{A}\left(\left(S^{*} \cup T\right) \cup\{0\}\right)-v_{A}\left(\left(\left(S^{*} \cup T\right) \backslash\{t\}\right) \cup\{0\}\right)>0 .
\end{aligned}
$$


Take any $\mu_{1} \in \mathcal{M}_{A}\left(S^{*} \cup\{t\}, Q\right)$. If $\mu_{1} \in \mathcal{M}_{A}^{s_{-t}}\left(S^{*} \cup\{t\}, Q\right)$, take $\bar{P} \in \mathcal{P}\left(\mu_{1}, S^{*}\right)$ as in expression (19). Therefore, $U_{i}\left(\mu_{1}, \bar{P}\right)=U_{i}\left(\mu, P^{*}\right)$ for all $i \in S^{*}$ and we have

$$
\begin{aligned}
\sum_{i \in S^{*}} P_{\mu(i)}^{*} & =v_{A}\left(S^{*} \cup\{0\}\right)-\sum_{i \in S^{*}} U_{i}\left(\mu, P^{*}\right) \\
& <v_{A}\left(\left(S^{*} \cup\{t\}\right) \cup\{0\}\right)-\sum_{i \in S^{*}} U_{i}\left(\mu_{1}, \bar{P}\right)=\sum_{i \in S^{*}} \bar{P}_{\mu_{1}(i)}+\sum_{j \in \mu_{1}(t)} a_{t j},
\end{aligned}
$$

which contradicts condition $(c)$ of Proposition 5.1 and the fact that $(s, \mathcal{O}(s))$ is a SPE. As a consequence $\mu_{1} \notin \mathcal{M}_{A}^{s-t}\left(S^{*} \cup\{t\}, Q\right)$.

By applying Lemma A.1, see Appendix A, to $S=S^{*}$ and $T^{\prime}=S^{*} \cup\{t\}$, there exist $\emptyset \neq T \varsubsetneqq T^{\prime}$ with $t \in T$ and $\mu^{\prime} \in \mathcal{M}_{A}^{s-t}(T, Q)$ such that

$$
\begin{aligned}
\sum_{i \in S^{*} \backslash T}\left(\sum_{j \in R(i)} a_{i j}-P_{R(i)}\right) & >v_{A}\left(\left(S^{*} \cup\{t\}\right) \cup\{0\}\right)-v_{A}(T \cup\{0\}) \\
& >v_{A}\left(S^{*} \cup\{0\}\right)-v_{A}(T \cup\{0\}),
\end{aligned}
$$

where $\left(R(i), P_{R(i)}\right)=s_{i}$ for all $i \in S^{*} \backslash T$, the second inequality comes from (21) taking $S=S^{*} \cup\{t\}$. Moreover, notice that

$$
\sum_{i \in S^{*} \backslash T} U_{i}\left(\mu, P^{*}\right)=\sum_{i \in S^{*} \backslash T}\left(\sum_{j \in R(i)} a_{i j}-P_{R(i)}\right)>v_{A}\left(S^{*} \cup\{0\}\right)-v_{A}(T \cup\{0\}) .
$$

Consider $\mu^{\prime} \in \mathcal{M}_{A}^{s-t}(T, Q)$ and take $\bar{P}^{\prime} \in \mathcal{P}\left(\mu^{\prime}, T \backslash\{t\}\right)$ as in expression (19). Therefore, $U_{i}\left(\mu^{\prime}, \bar{P}^{\prime}\right)=U_{i}\left(\mu, P^{*}\right)$ for all $i \in T \cap S^{*}$. Taking (22) into account, we get

$$
\begin{aligned}
\sum_{i \in S^{*}} P_{\mu(i)}^{*} & =v_{A}\left(S^{*} \cup\{0\}\right)-\sum_{i \in S^{*}} U_{i}\left(\mu, P^{*}\right) \\
& <v_{A}(T \cup\{0\})-\sum_{i \in T \backslash\{t\}} U_{i}\left(\mu^{\prime}, \bar{P}^{\prime}\right)=\sum_{i \in T \backslash\{t\}} P_{\mu(i)}^{\prime}+\sum_{j \in \mu(t)} a_{t j},
\end{aligned}
$$

which contradicts condition $(c)$ of Proposition 5.1 (recall that $t \in M \backslash S^{*}$ ). Hence $v_{A}\left(S^{*} \cup\{0\}\right)=v_{A}(M \cup\{0\})$.

The following remark will be used later on. It shows that in a SPE, buyers not selected by the seller get their marginal contributions.

Remark 5.4. Let $(s, \mathcal{O}(s))$ be a SPE of $\Gamma$ where $\mathcal{O}(s)$ is formed by the triple $\left(S^{*}, \mu, P^{*}\right)$. As a consequence of Proposition 5.3 and monotonicity of $v_{A}, M_{i}^{v_{A}}=0$ for each buyer $i \in M \backslash S^{*}$. Indeed, $U_{i}\left(\mu, P^{*}\right)=0=M_{i}^{v_{A}}$ for all $i \in M \backslash S^{*}$.

Now, let us introduce some notation.

Definition 5.5. Let $(M,\{0\}, Q, A, r)$ be a one-seller assignment market with multi-unit demands and $(s, \mathcal{O}(s))$ be a SPE of $\Gamma$ where $\mathcal{O}(s)$ is formed by the triple $\left(S^{*}, \mu, P^{*}\right)$. For each $i^{\prime} \in S^{*}$, we denote by:

$$
S^{i^{\prime}} \subseteq M \backslash\left\{i^{\prime}\right\}, \mu^{i^{\prime}} \in \mathcal{M}^{s}\left(S^{i^{\prime}}, Q\right) \text { and } P^{i^{\prime}} \in \mathcal{P}\left(\mu^{i^{\prime}}, S^{i^{\prime}}\right)
$$


the coalition, the matching and the price vector that satisfy condition (b) of Proposition 5.1. That is

$$
\sum_{i \in S^{*}} P_{\mu(i)}^{*}=\sum_{i \in S^{i^{\prime}}} P_{\mu^{i^{\prime}}(i)}^{i^{\prime}}
$$

Notice that, in the $\operatorname{SPE}(s, \mathcal{O}(s))$, if we take any $i^{\prime} \in S^{*}$, we know that $U_{i}\left(\mu, P^{*}\right)=0$ for all $i \in S^{i^{\prime}} \backslash S^{*}$. Now, we claim that

$$
\sum_{j \in R(i)} a_{i j}-P_{R(i)}=U_{i}\left(\mu^{i^{\prime}}, P^{i^{\prime}}\right)=0 \text { for all } i \in S^{i^{\prime}} \backslash S^{*},
$$

where $\left(R(i), P_{R(i)}\right)=s_{i}$. Otherwise, if for some $i \in S^{i^{\prime}} \backslash S^{*}, \sum_{j \in R(i)} a_{i j}-P_{R(i)}>0$, this buyer would have incentives to increase a bit $P_{R(i)}$ to force the seller to choose $\mu^{i^{\prime}}$ instead of $\mu$ in order to get a positive a positive payoff. Moreover, by condition (b) of Proposition (5.1), we have

$$
\sum_{i \in S^{*}} P_{\mu(i)}^{*}=\sum_{i \in S^{i^{\prime}}} P_{\mu^{i^{\prime}}(i)}^{i^{\prime}}
$$

Therefore, we claim that $P^{i^{\prime}} \in \mathcal{P}\left(\mu^{i^{\prime}}, S^{i^{\prime}}\right)$ satisfies

$$
\sum_{j \in \mu^{i^{\prime}}(i)} a_{i j}-P_{\mu^{i^{\prime}(i)}}^{i^{\prime}}=\sum_{j \in R(i)} a_{i j}-P_{R(i)} \text { for all } i \in S^{i^{\prime}}
$$

where $\left(R(i), P_{R(i)}\right)=s_{i}$. Otherwise, if for some $i^{*} \in S^{i^{\prime}}$, it holds that $\sum_{j \in \mu^{i^{\prime}\left(i^{*}\right)}} a_{i^{*} j}-$ $P_{\mu^{i^{\prime}\left(i^{*}\right)}}^{i^{\prime}}>\sum_{j \in R\left(i^{*}\right)} a_{i^{*} j}-P_{R\left(i^{*}\right)}$, take $\bar{P}^{i^{\prime}} \in \mathcal{P}\left(\mu^{i^{\prime}}, S^{i^{\prime}}\right)$ as in expression (14). Notice that

$$
\sum_{i \in S^{*}} P_{\mu(i)}^{*}=\sum_{i \in S^{i^{\prime}}} P_{\mu^{i^{\prime}(i)}}^{i^{\prime}}<\sum_{i \in S^{i^{\prime}}} \bar{P}_{\mu^{i^{\prime}(i)}}^{i^{\prime}}
$$

this contradicts condition (a) of Proposition (5.1).

Now, as the main result of this section, we prove that the unique payoff vector of all $\mathrm{SPE}$ of $\Gamma$ is the buyers-optimal core allocation.

Theorem 5.6. Let $(M,\{0\}, Q, A, r)$ be a one-seller assignment market with multi-unit demands. The outcome of any SPE of $\Gamma$ is the buyers-optimal core allocation.

Proof. Take any SPE $(s, \mathcal{O}(s))$, where $\mathcal{O}(s)$ is formed by the triple $\left(S^{*}, \mu, P^{*}\right)$. We first prove that for any $i^{\prime} \in S^{*}$, there is some $\mu \in \mathcal{M}_{A}^{s}\left(\left(S^{*} \cup S^{i^{\prime}}\right) \backslash\left\{i^{\prime}\right\}, Q\right)$ where $S^{i^{\prime}}$ is the coalition introduced in Definition 5.5. To this end, assume on the contrary that $(s, \mathcal{O}(s))$ is a SPE and for all $\mu \in \mathcal{M}_{A}\left(\left(S^{*} \cup S^{i^{\prime}}\right) \backslash\left\{i^{\prime}\right\}, Q\right)$, we have $\mu \notin \mathcal{M}_{A}^{s}\left(\left(S^{*} \cup S^{i^{\prime}}\right) \backslash\left\{i^{\prime}\right\}, Q\right)$. This implies that there is some $t \in\left(S^{*} \cup S^{i^{\prime}}\right) \backslash\left\{i^{\prime}\right\}$ such that

$$
\begin{aligned}
\sum_{j \in R(t)} a_{t j}-P_{R(t)} & >\sum_{j \in \mu(t)} a_{t j} \\
& \geq v_{A}\left(\left(\left(S^{*} \cup S^{i^{\prime}}\right) \backslash\left\{i^{\prime}\right\}\right) \cup\{0\}\right)-v_{A}\left(\left(\left(S^{*} \cup S^{i^{\prime}}\right) \backslash\left\{t, i^{\prime}\right\}\right) \cup\{0\}\right) \\
& \geq v_{A}\left(\left(S^{*} \cup S^{i^{\prime}}\right) \cup\{0\}\right)-v_{A}\left(\left(\left(S^{*} \cup S^{i^{\prime}}\right) \backslash\{t\}\right) \cup\{0\}\right),
\end{aligned}
$$


where $\left(R(t), P_{R(t)}\right)=s_{t}$, the last inequality follows from buyers-submodular condition (2). By expression (23), the above strict inequality implies that $t \in S^{*}$. Taking that into account, we prove that if buyer $t$ requests $\left(R(t), P_{R(t)}\right)$ such that $\sum_{j \in R(t)} a_{t j}-P_{R(t)}>$ $\sum_{j \in \mu(t)} a_{t j}$, we will reach a contradiction with buyer t belonging to $S^{*}$.

Take any $\mu_{1} \in \mathcal{M}_{A}\left(\left(S^{*} \cup S^{i^{\prime}}\right) \backslash\{t\}, Q\right)$. If $\mu_{1} \in \mathcal{M}_{A}^{s}\left(\left(S^{*} \cup S^{i^{\prime}}\right) \backslash\{t\}, Q\right)$, take $\bar{P} \in \mathcal{P}\left(\mu_{1},\left(S^{*} \cup S^{i^{\prime}}\right) \backslash\{t\}\right)$ as in expression (19). Therefore, $U_{i}\left(\mu_{1}, \bar{P}\right)=U_{i}\left(\mu, P^{*}\right)$ for all $i \in\left(S^{*} \cup S^{i^{\prime}}\right) \backslash\{t\}$ and we have

$$
\begin{aligned}
\sum_{i \in S^{*}} P_{\mu(i)}^{*} & =v_{A}(M \cup\{0\})-\sum_{i \in S^{*}} U_{i}\left(\mu, P^{*}\right)=v_{A}(M \cup\{0\})-\sum_{i \in S^{*} \cup S^{i^{\prime}}} U_{i}\left(\mu, P^{*}\right) \\
& =v_{A}\left(\left(S^{*} \cup S^{i^{\prime}}\right) \cup\{0\}\right)-\sum_{i \in S^{*} \cup S^{i^{\prime}}} U_{i}\left(\mu, P^{*}\right) \\
& <v_{A}\left(\left(\left(S^{*} \cup S^{i^{\prime}}\right) \backslash\{t\}\right) \cup\{0\}\right)-\sum_{i \in\left(S^{*} \cup S^{i^{\prime}}\right) \backslash\{t\}} U_{i}\left(\mu_{1}, \bar{P}\right) \\
& =\sum_{i \in\left(S^{*} \cup S^{i^{\prime}}\right) \backslash\{t\}} \bar{P}_{\mu_{1}(i)},
\end{aligned}
$$

where the first inequality follows from Proposition 5.3, the second one from $U_{i}\left(\mu, P^{*}\right)=0$ for all $i \in S^{i^{\prime}} \backslash S^{*}$, the third one because of monotonicity of $v_{A}$ and the inequality from (26). This contradicts (16) and the fact that $(s, \mathcal{O}(s))$ is a SPE. As a consequence $\left.\mu_{1} \notin \mathcal{M}_{A}^{s}\left(S^{*} \cup S^{i^{\prime}}\right) \backslash\{t\}, Q\right)$.

By applying Lemma A.1, see Appendix A, to $S=T^{\prime}=\left(S^{*} \cup S^{i^{\prime}}\right) \backslash\{t\}$, there exists $\emptyset \neq T \varsubsetneqq T^{\prime}$ and $\mu^{\prime} \in \mathcal{M}_{A}^{s}(T, Q)$, such that

$$
\sum_{i \in\left(\left(S^{*} \cup S^{i^{\prime}}\right) \backslash\{t\}\right) \backslash T}\left(\sum_{j \in R(i)} a_{i j}-P_{R(i)}\right)>v_{A}\left(\left(\left(S^{*} \cup S^{i^{\prime}}\right) \backslash\{t\}\right) \cup\{0\}\right)-v_{A}(T \cup\{0\}) .
$$

where $\left(R(i), P_{R(i)}\right)=s_{i}$ for all $i \in\left(\left(S^{*} \cup S^{i^{\prime}}\right) \backslash\{t\}\right) \backslash T$. Making use of (26) notice that,

$$
\begin{aligned}
\sum_{i \in\left(S^{*} \cup S^{i^{\prime}}\right) \backslash T} & \left(\sum_{j \in R(i)} a_{i j}-P_{R(i)}\right)>v_{A}\left(\left(\left(S^{*} \cup S^{i^{\prime}}\right) \backslash\{t\}\right) \cup\{0\}\right)-v_{A}(T \cup\{0\}) \\
& +v_{A}\left(\left(S^{*} \cup S^{i^{\prime}}\right) \cup\{0\}\right)-v_{A}\left(\left(\left(S^{*} \cup S^{i^{\prime}}\right) \backslash\{t\}\right) \cup\{0\}\right) \\
& =v_{A}\left(\left(S^{*} \cup S^{i^{\prime}}\right) \cup\{0\}\right)-v_{A}(T \cup\{0\})
\end{aligned}
$$

where $\left(R(i), P_{R(i)}\right)=s_{i}$ for all $i \in\left(S^{*} \cup S^{i^{\prime}}\right) \backslash T$.

Consider $\mu^{\prime} \in \mathcal{M}_{A}^{s}(T, Q)$ and take $\bar{P}^{\prime} \in \mathcal{P}\left(\mu^{\prime}, T\right)$ as in expression (19). Therefore, $U_{i}\left(\mu^{\prime}, \bar{P}^{\prime}\right)=U_{i}\left(\mu, P^{*}\right)$ for all $i \in T$ and we have

$$
\begin{aligned}
v_{A}\left(\left(S^{*} \cup S^{i^{\prime}}\right) \cup\{0\}\right)-\sum_{i \in S^{*} \cup S^{i^{\prime}}} U_{i}\left(\mu, P^{*}\right) & =v_{A}(M \cup\{0\})-\sum_{i \in S^{*} \cup S^{i^{\prime}}} U_{i}\left(\mu, P^{*}\right) \\
& =v_{A}(M \cup\{0\})-\sum_{i \in S^{*}} U_{i}\left(\mu, P^{*}\right)=\sum_{i \in S^{*}} P_{\mu(i)}^{*} \\
& \geq \sum_{i \in T} \bar{P}_{\mu^{\prime}(i)}^{\prime}=v_{A}(T \cup\{0\})-\sum_{i \in T} U_{i}\left(\mu^{\prime}, \bar{P}^{\prime}\right),
\end{aligned}
$$


where the first equality comes from monotonicity of $v_{A}$, the second one because of (5.5), the third equality follow from Proposition 5.3 and the inequality from condition $(a)$ of SPE in Proposition 5.1. Then,

$$
v_{A}\left(\left(S^{*} \cup S^{i^{\prime}}\right) \cup\{0\}\right)-v_{A}(T \cup\{0\}) \geq \sum_{i \in S^{*} \backslash T} U_{i}\left(\mu, P^{*}\right)=\sum_{i \in\left(S^{*} \cup S^{i^{\prime}}\right) \backslash T}\left(\sum_{j \in R(i)} a_{i j}-P_{R(i)}\right),
$$

where $\left(R(i), P_{R(i)}\right)=s_{i}$ for all $i \in\left(S^{*} \cup S^{i^{\prime}}\right) \backslash T$. This is a contradiction with (27) and hence there is some matching $\mu \in \mathcal{M}_{A}^{s}\left(\left(S^{*} \cup S^{i^{\prime}}\right) \backslash\left\{i^{\prime}\right\}, Q\right)$ and the claim is proved.

Now, we prove that the outcome of $(s, \mathcal{O}(s))$ is the buyers-optimal core allocation. We have seen that for each $i \in S^{*}$ there exists $\mu \in \mathcal{M}_{A}^{s}\left(\left(S^{*} \cup S^{i}\right) \backslash\{i\}, Q\right)$. Take a buyer $i^{\prime} \in S^{*}$. Consider $\mu^{\prime} \in \mathcal{M}_{A}^{s}\left(\left(S^{*} \cup S^{i^{\prime}}\right) \backslash\left\{i^{\prime}\right\}, Q\right)$ and $\bar{P}^{\prime} \in \mathcal{P}\left(\mu^{\prime},\left(S^{*} \cup S^{i^{\prime}}\right) \backslash\left\{i^{\prime}\right\}\right)$ as in expression (19). Therefore, $U_{i}\left(\mu^{\prime}, \bar{P}^{\prime}\right)=U_{i}\left(\mu, P^{*}\right)$ for all $i \in\left(S^{*} \cup S^{i^{\prime}}\right) \backslash\left\{i^{\prime}\right\}$ and we have

$$
\begin{aligned}
& v_{A}(M \cup\{0\})-\sum_{i \in S^{*}} U_{i}\left(\mu, P^{*}\right)=\sum_{i \in S^{*}} P_{\mu(i)}^{*} \\
& \geq \sum_{i \in\left(S^{*} \cup S^{i^{\prime}}\right) \backslash\left\{i^{\prime}\right\}} \bar{P}_{\mu^{\prime}(i)}^{\prime}=v_{A}\left(\left(M \backslash\left\{i^{\prime}\right\}\right) \cup\{0\}\right)-\sum_{i \in\left(S^{*} \cup S^{i^{\prime}}\right) \backslash\left\{i^{\prime}\right\}} U_{i}\left(\mu^{\prime}, \bar{P}^{\prime}\right),
\end{aligned}
$$

where the first equality follows from Proposition 5.3, the inequality from condition $(a)$ of Proposition 5.1, and the last equality from Proposition A.3 (in Appendix A). Then,

$$
v_{A}(M \cup\{0\})-v_{A}\left(\left(M \backslash\left\{i^{\prime}\right\}\right) \cup\{0\}\right) \geq \sum_{i \in S^{*}} U_{i}\left(\mu, P^{*}\right)-\sum_{i \in\left(S^{*} \cup S^{i^{\prime}}\right) \backslash\left\{i^{\prime}\right\}} U_{i}\left(\mu^{\prime}, \bar{P}^{\prime}\right) .
$$

Since $U_{i}\left(\mu, P^{*}\right)=U_{i}\left(\mu^{\prime}, \bar{P}^{\prime}\right)$ for all $i \in S^{*} \cap S^{i^{\prime}}$ and, by Remark 5.4, $U_{i}\left(\mu, P^{*}\right)=0$ for all $i \in S^{i^{\prime}} \backslash S^{*}$, we get

$$
v_{A}(M \cup\{0\})-v_{A}\left(\left(M \backslash\left\{i^{\prime}\right\}\right) \cup\{0\}\right) \geq U_{i^{\prime}}\left(\mu, P^{*}\right) .
$$

By Proposition 5.3 and Remark 5.4, we know that in any $\operatorname{SPE}(s, \mathcal{O}(s)), \sum_{(i, j) \in \mu} a_{i j}=$ $v_{A}(M \cup\{0\})$ and $U_{i}\left(\mu, P^{*}\right)=M_{i}^{v_{A}}$ for all $i \in M \backslash S^{*}$. Moreover, by expression (28), $M_{i}^{v_{A}} \geq U_{i}\left(\mu, P^{*}\right)$ for all $i \in S^{*}$. Hence, we must see that $U_{i}\left(\mu, P^{*}\right) \leq M_{i}^{v_{A}}$ for all $i \in S^{*}$.

Take a buyer $i^{\prime} \in S^{*}$, let $S^{i^{\prime}}$ and $\mu^{i^{\prime}} \in \mathcal{M}^{s}\left(S^{i^{\prime}}, Q\right)$ be as in Definition 5.5 and take $P^{i^{\prime}} \in \mathcal{P}\left(\mu^{i^{\prime}}, S^{i^{\prime}}\right)$, then

$$
\begin{aligned}
v_{A}\left(S^{*} \cup\{0\}\right)-\sum_{i \in S^{*}} U_{i}\left(\mu, P^{*}\right) & =\sum_{i \in S^{*}} P_{\mu(i)}^{*} \\
& =\sum_{i \in S^{i^{\prime}}} P_{\mu^{i^{\prime}}(i)}^{i^{\prime}}=v_{A}\left(S^{i^{\prime}} \cup\{0\}\right)-\sum_{i \in S^{i^{\prime}}} U_{i}\left(\mu^{i^{\prime}}, P^{i^{\prime}}\right),
\end{aligned}
$$

where the last equality follows from Proposition A.2, see Appendix A. As a consequence,

$$
v_{A}\left(S^{*} \cup\{0\}\right)-v_{A}\left(S^{i^{\prime}} \cup\{0\}\right)=\sum_{i \in S^{*} \backslash S^{i^{\prime}}} U_{i}\left(\mu, P^{*}\right)-\sum_{i \in S^{i^{\prime}} \backslash S^{*}} U_{i}\left(\mu^{i^{\prime}}, P^{i^{\prime}}\right) .
$$


By expression (23), we know that $U_{i}\left(\mu^{i^{\prime}}, P^{i^{\prime}}\right)=0$ for all $i \in S^{i^{\prime}} \backslash S^{*}$. Therefore

$$
v_{A}\left(S^{*} \cup\{0\}\right)-v_{A}\left(S^{i^{\prime}} \cup\{0\}\right)=\sum_{i \in S^{*} \backslash S^{i^{\prime}}} U_{i}\left(\mu, P^{*}\right) .
$$

On the other hand, since $v_{A}\left(S^{*} \cup\{0\}\right)=v_{A}(M \cup\{0\})$ and monotonicity of $v_{A}$, we have that $v_{A}\left(\left(S^{*} \cup S^{i^{\prime}}\right) \cup\{0\}\right)=v_{A}\left(S^{*} \cup\{0\}\right)$. Then, by buyers-submodularity (??) and (2), we get

$$
\begin{aligned}
& v_{A}\left(S^{*} \cup\{0\}\right)-v_{A}\left(S^{i^{\prime}} \cup\{0\}\right)=v_{A}\left(\left(S^{*} \cup S^{i^{\prime}}\right) \cup\{0\}\right)-v_{A}\left(S^{i^{\prime}} \cup\{0\}\right) \\
& \geq \sum_{i \in S^{*} \backslash S^{i^{\prime}}}\left(v_{A}\left(\left(S^{*} \cup S^{i^{\prime}}\right) \cup\{0\}\right)-v_{A}\left(\left(\left(S^{*} \cup S^{i^{\prime}}\right) \backslash\{i\}\right) \cup\{0\}\right)\right) \geq \sum_{i \in S^{*} \backslash S^{i^{\prime}}} M_{i}^{v_{A}} .
\end{aligned}
$$

Then, making use of (29)

$$
\sum_{i \in S^{*} \backslash S^{i^{\prime}}} U_{i}\left(\mu, P^{*}\right) \geq \sum_{i \in S^{*} \backslash S^{i^{\prime}}} M_{i}^{v_{A}} .
$$

Since we have $U_{i}\left(\mu, P^{*}\right) \leq M_{i}^{v_{A}}$ for all $i \in S^{*}$, we get $M_{i}^{v_{A}}=U_{i}\left(\mu, P^{*}\right)$ for all $i \in S^{*} \backslash S^{i^{\prime}}$. This completes the proof: in any $\operatorname{SPE}(s, \mathcal{O}(s))$., the payoff vector is $U_{i}\left(\mu, P^{*}\right)=M_{i}^{v_{A}}$ for each $i \in M$ and $V\left(\mu, P^{*}\right)=v_{A}(M \cup\{0\})-\sum_{i \in M} M_{i}^{v_{A}}$ for the seller, which is the buyers-optimal core allocation.

Therefore, we have proved that in every SPE, each buyer receives his maximum core payoff, that is his marginal contribution, while the seller gets her minimum one, $v_{A}(M \cup\{0\})-\sum_{i \in M} M_{i}^{v_{A}}$. Hence the buyers-optimal core allocation is the outcome of every SPE of the mechanism $\Gamma$.

\section{Concluding Remarks}

In Tauman et al. (1997), it is considered a somehow related many-to-one model with only one buyer with multi-unit demand but several sellers with unitary supply. The valuation function of the buyer is not necessary linear and a strategic form game is provided. This strategic game leads to the outcome where each seller gets her marginal contribution by means of the subgame perfect Nash equilibrium. The authors mention that an important and nontrivial extension of their model would be the case where firms produce more than one product. To some extent, this is what our model provides, although the sector with only one agent is now the one of the sellers to lie in the setting of the Vickrey auction.

As a drawback, we remain in the case in which, buyers' valuations of packages are linear. We believe that this fact, which implies the buyers-subadditive property, is crucial to obtain, differently from the aforementioned model, the buyers-optimal core allocation as the unique subgame perfect Nash equilibrium outcome. 


\section{A Appendix}

Lemma A.1. Let $s$ be a buyers' strategy profile of procedure $\Gamma$ and $\emptyset \neq S \subseteq M$ be a coalition of buyers. For all $T^{\prime} \subseteq M$ such that $S \cap T^{\prime} \neq \emptyset$, there exists $T \subseteq T^{\prime}$ with $T^{\prime} \backslash S \subseteq T$ and a matching $\mu \in \overline{\mathcal{M}}_{A}^{s_{-\left(T^{\prime} \backslash S\right)}}(T, Q)$. If $T \neq T^{\prime}$, it holds that

$$
\sum_{i \in\left(T^{\prime} \cap S\right) \backslash T}\left(\sum_{j \in R(i)} a_{i j}-P_{R(i)}\right)>v_{A}\left(T^{\prime} \cup\{0\}\right)-v_{A}(T \cup\{0\}),
$$

where $\left(R(i), P_{R(i)}\right)=s_{i}$ for each $i \in\left(T^{\prime} \cap S\right) \backslash T$.

Proof. Take any coalition of buyers $\emptyset \neq S \subseteq M$ and $T^{\prime} \subseteq M$ such that $S \cap T^{\prime} \neq \emptyset$. First, consider $T_{1}=T^{\prime}$ and $\mu_{1} \in \mathcal{M}_{A}\left(T^{\prime}, Q\right)$. If $\mu_{1} \in \mathcal{M}_{A}^{s-\left(T^{\prime} \backslash S\right)}\left(T^{\prime}, Q\right)$, we are done just taking $T=T_{1}=T^{\prime}$. Otherwise, if $\mu_{1} \notin \mathcal{M}_{A}^{s-\left(T^{\prime} \backslash S\right)}\left(T^{\prime}, Q\right)$ there exists some $i_{1} \in T_{1} \cap S$ such that

$$
\sum_{j \in R\left(i_{1}\right)} a_{i_{1} j}-P_{R\left(i_{1}\right)}>\sum_{j \in \mu_{1}\left(i_{1}\right)} a_{i_{1} j}
$$

where $\left(R\left(i_{1}\right), P_{R\left(i_{1}\right)}\right)=s_{i_{1}}$. By the optimality of $\mu_{1}$, we have

$$
\sum_{j \in R\left(i_{1}\right)} a_{i_{1} j}-P_{R\left(i_{1}\right)}>\sum_{j \in \mu_{1}\left(i_{1}\right)} a_{i_{1} j} \geq v_{A}\left(T^{\prime} \cup\{0\}\right)-v_{A}\left(\left(T^{\prime} \backslash\left\{i_{1}\right\}\right) \cup\{0\}\right) .
$$

Take now any $\mu_{2} \in \mathcal{M}_{A}\left(T^{\prime} \backslash\left\{i_{1}\right\}, Q\right)$. If $\mu_{2} \in \mathcal{M}_{A}^{s-\left(T^{\prime} \backslash S\right)}\left(T^{\prime} \backslash\left\{i_{1}\right\}, Q\right)$, we are done with $T=T_{2}=T^{\prime} \backslash\left\{i_{1}\right\}$. Otherwise, if $\mu_{2} \notin \mathcal{M}_{A}^{s_{-\left(T^{\prime} \backslash S\right)}}\left(T^{\prime} \backslash\left\{i_{1}\right\}, Q\right)$, then there exists some buyer $i_{2} \in T_{2} \cap S$ such that

$$
\sum_{j \in R\left(i_{2}\right)} a_{i_{2} j}-P_{R\left(i_{1}\right)}>\sum_{j \in \mu_{2}\left(i_{2}\right)} a_{i_{2} j} \geq v_{A}\left(T_{2} \cup\{0\}\right)-v_{A}\left(T_{3} \cup\{0\}\right),
$$

where $\left(R\left(i_{2}\right), P_{R\left(i_{2}\right)}\right)=s_{i_{2}}$ and $T_{3}=T_{2} \backslash\left\{i_{2}\right\}$. By adding (30) and (31) we get

$$
\sum_{i \in\left(T^{\prime} \cap S\right) \backslash T_{3}}\left(\sum_{j \in R(i)} a_{i j}-P_{R\left(i_{1}\right)}\right)>v_{A}\left(T^{\prime} \cup\{0\}\right)-v_{A}\left(T_{3} \cup\{0\}\right),
$$

where $\left(R(i), P_{R(i)}\right)=s_{i}$ for all $i \in\left(T^{\prime} \cap S\right) \backslash T_{3}$.

By proceeding recursively, assume we have constructed a sequence $\left\{i_{1}, \ldots, i_{k}\right\} \subseteq T^{\prime} \cap S$ such that for all $l \in\{1, \ldots, k\}$ it holds $\mathcal{M}_{A}^{s_{-\left(T^{\prime} \backslash S\right)}}\left(T^{\prime} \backslash\left\{i_{1}, \ldots, i_{l}\right\}, Q\right)=\emptyset$ and if we write $T_{k+1}=T^{\prime} \backslash\left\{i_{1}, \ldots, i_{k}\right\}$ it holds

$$
\sum_{i \in\left(T^{\prime} \cap S\right) \backslash T_{k+1}}\left(\sum_{j \in R(i)} a_{i j}-P_{R(i)}\right)>v_{A}\left(T^{\prime} \cup\{0\}\right)-v_{A}\left(T_{k+1} \cup\{0\}\right),
$$


where $\left(R_{i}, P_{R(i)}\right)=s_{i}$ for all $i \in\left(T^{\prime} \cap S\right) \backslash T_{k+1}$. So, either $\mathcal{M}_{A}^{s_{-\left(T^{\prime} \backslash S\right)}}\left(T_{k+1}, Q\right) \neq \emptyset$ and we are done with $T=T_{k+1}$ or take $\mu_{k+1} \in \mathcal{M}_{A}\left(T_{k+1}, Q\right)$. Then there exists some buyer $i_{k+1} \in T_{k+1} \cap S$ such that,

$\sum_{j \in R\left(i_{k+1}\right)} a_{i_{k+1} j}-P_{R\left(i_{k+1}\right)}>\sum_{j \in \mu_{k+1}\left(i_{k+1}\right)} a_{i_{k+1} j} \geq v_{A}\left(T_{k+1} \cup\{0\}\right)-v_{A}\left(\left(T_{k+1} \backslash\left\{i_{k+1}\right\}\right) \cup\{0\}\right)$,

where $\left(R\left(i_{k+1}\right), P_{R\left(i_{k+1}\right)}\right)=s_{i_{k+1}}$. By combining (32) and (33), we define $T_{k+2}=T_{k+1} \backslash$ $\left\{i_{k+1}\right\}$ and obtain

$$
\sum_{i \in\left(T^{\prime} \cap S\right) \backslash T_{k+2}}\left(\sum_{j \in R_{i}} a_{i j}-P^{i}\left(R_{i}\right)\right)>v_{A}\left(T^{\prime} \cup\{0\}\right)-v_{A}\left(T_{k+2} \cup\{0\}\right),
$$

where $\left(R_{i}, P_{R(i)}\right)=s_{i}$ for all $i \in\left(T^{\prime} \cap S\right) \backslash T_{k+2}$

Now either there exists $\mu \in \mathcal{M}_{A}^{s_{-\left(T^{\prime} \backslash S\right)}}\left(T_{k+2}, Q\right)$ where $T_{k+2} \varsubsetneqq T_{k+1}$ and we are done with $T=T_{k+2}$ or we continue the procedure one more step. Notice that, since $T^{\prime} \cap S$ is finite, if it does not stop before, the procedure will reach $T_{k}$ with $\left|T_{k}\right|=1$ for some $k \geq 1$. In that case, let us write $T_{k}=\{i\}$. If $\mu_{k} \in \mathcal{M}_{A}\left(T_{k}, Q\right)$ then

$$
\sum_{j \in \mu_{k}(i)} a_{i j} \geq \sum_{j \in R(i)} a_{i j}-P_{R(i)}
$$

where $\left(R(i), P_{R(i)}\right)=s_{i}$, which means there exists $P_{\mu_{k}(i)} \in \mathbb{R}_{+}$that satisfies (15), hence $\mu_{k} \in \mathcal{M}_{A}^{s_{-\left(T^{\prime} \backslash S\right)}}\left(T_{k}, Q\right)$ and we are done with $T=T_{k}$.

Proposition A.2. Let $(M,\{0\}, Q, A, r)$ be a one-seller assignment market with multiunit demands and $(s, \mathcal{O}(s))$ be a SPE of $\Gamma$, where $\mathcal{O}(s)$ is formed by the triple $\left(S^{*}, \mu, P^{*}\right)$. For any $i^{\prime} \in S^{*}$, let $S^{i^{\prime}}$ be the coalition and $\mu^{i^{\prime}} \in \mathcal{M}^{s}\left(S^{i^{\prime}}, Q\right)$ be the matching as introduced in Definition 5.5. Then

$$
\sum_{(i, j) \in \mu^{i^{\prime}}} a_{i j}=v_{A}\left(S^{i^{\prime}} \cup\{0\}\right)
$$

Proof. Assume on the contrary that $(s, \mathcal{O}(s))$ is a SPE and $\sum_{(i, j) \in \mu^{i^{\prime}}} a_{i j}<v_{A}\left(S^{i^{\prime}} \cup\{0\}\right)$. Take any $\mu_{1} \in \mathcal{M}_{A}\left(S^{i^{\prime}}, Q\right)$. If $\mu_{1} \in \mathcal{M}_{A}^{s}\left(S^{i^{\prime}}, Q\right)$, take $\bar{P} \in \mathcal{P}\left(\mu_{1}, S^{i^{\prime}}\right)$ as in expression (19). Therefore, because of $(25), U_{i}\left(\mu_{1}, \bar{P}\right)=U_{i}\left(\mu^{i^{\prime}}, P^{i^{\prime}}\right)$ for all $i \in S^{i^{\prime}}$ and making use of condition (b), we have

$$
\begin{aligned}
\sum_{i \in S^{*}} P_{\mu(i)}^{*}=\sum_{i \in S^{i^{\prime}}} P_{\mu^{i^{\prime}}(i)}^{i^{\prime}} & =\sum_{(i, j) \in \mu^{i^{\prime}}} a_{i j}-\sum_{i \in S^{i^{\prime}}} U_{i}\left(\mu^{i^{\prime}}, P^{i^{\prime}}\right) \\
& <v_{A}\left(S^{i^{\prime}} \cup\{0\}\right)-\sum_{i \in S^{i^{\prime}}} U_{i}\left(\mu_{1}, \bar{P}\right)=\sum_{i \in S^{i^{\prime}}} \bar{P}_{\mu_{1}(i)},
\end{aligned}
$$

which is a contradiction. Therefore $\mu_{1} \notin \mathcal{M}_{A}^{s}\left(S^{i^{\prime}}, Q\right)$. 
By applying Lemma A.1, in this Appendix, to $S=T^{\prime}=S^{i^{\prime}}$, there exists $\emptyset \neq T \varsubsetneqq T^{\prime}$ and $\mu^{\prime} \in \mathcal{M}_{A}^{s}(T, Q)$ such that

$$
\sum_{i \in S^{i^{\prime}} \backslash T}\left(\sum_{j \in R(i)} a_{i j}-P_{R(i)}\right)>v_{A}\left(S^{i^{\prime}} \cup\{0\}\right)-v_{A}(T \cup\{0\}),
$$

where $\left(R(i), P_{R(i)}\right)=s_{i}$ for all $i \in S^{i^{\prime}} \backslash T$.

Consider $\mu^{\prime} \in \mathcal{M}_{A}^{s}(T, Q)$ and take $\bar{P}^{\prime} \in \mathcal{P}\left(\mu^{\prime}, T\right)$ as in expression (19). Therefore, $U_{i}\left(\mu^{i^{\prime}}, P^{i^{\prime}}\right)=U_{i}\left(\mu^{\prime}, \bar{P}^{\prime}\right)$ for all $i \in T$. By conditions $(b)$ and $(a)$ in Proposition 5.1, we have

$$
\sum_{i \in S^{i^{\prime}}} P_{\mu^{i^{\prime}(i)}}^{i^{\prime}}=\sum_{i \in S^{*}} P_{\mu(i)}^{*} \geq \sum_{i \in T} \bar{P}_{\mu^{\prime}(i)}^{\prime}=v_{A}(T \cup\{0\})-\sum_{i \in T} U_{i}\left(\mu^{\prime}, \bar{P}^{\prime}\right)
$$

Hence

$$
\begin{aligned}
\sum_{(i, j) \in \mu^{i^{\prime}}} a_{i j}-v_{A}(T \cup\{0\}) & \geq \sum_{i \in S^{i^{\prime}}} U_{i}\left(\mu^{i^{\prime}}, P^{i^{\prime}}\right)-\sum_{i \in T} U_{i}\left(\mu, \bar{P}^{\prime}\right) \\
& =\sum_{i \in S^{i^{\prime}} \backslash T} U_{i}\left(\mu^{i^{\prime}}\right)=\sum_{i \in S^{i^{\prime}} \backslash T}\left(\sum_{j \in R_{i}} a_{i j}-P_{R(i)}^{i^{\prime}}\right),
\end{aligned}
$$

where $\left(R(i), P_{R\left(i_{1}\right)}\right)=s_{i}$ for all $i \in S^{i^{\prime}} \backslash T$. This contradicts $(34)$, hence $\sum_{(i, j) \in \mu^{i^{\prime}}} a_{i j}=$ $v_{A}\left(S^{i^{\prime}} \cup\{0\}\right)$.

Proposition A.3. Let $(M,\{0\}, Q, A, r)$ be a one-seller assignment market with multiunit demands and $(s, \mathcal{O}(s))$ be a $S P E$ of $\Gamma$, where $\mathcal{O}(s)$ is formed by the triple $\left(S^{*}, \mu, P^{*}\right)$. For any $i^{\prime} \in S^{*}$, let $S^{i^{\prime}}$ be the coalition introduced in Definition 5.5. Then

$$
v_{A}\left(\left(\left(S^{*} \cup S^{i^{\prime}}\right) \backslash\left\{i^{\prime}\right\}\right) \cup\{0\}\right)=v_{A}\left(\left(M \backslash\left\{i^{\prime}\right\}\right) \cup\{0\}\right) .
$$

Proof. First, we show that $v_{A}\left(\left(S^{i^{\prime}} \cup\{t\}\right) \cup\{0\}\right)=v_{A}\left(S^{i^{\prime}} \cup\{0\}\right)$ for any $t \in M \backslash\left(S^{*} \cup S^{i^{\prime}}\right)$. Assume on the contrary that $(s, \mathcal{O}(s))$ is a SPE and there is some $t \in M \backslash\left(S^{*} \cup S^{i^{\prime}}\right)$ such that $v_{A}\left(\left(S^{i^{\prime}} \cup\{t\}\right) \cup\{0\}\right)>v_{A}\left(S^{i^{\prime}} \cup\{0\}\right)$.

Consider any $\mu_{1} \in \mathcal{M}_{A}\left(S^{i^{\prime}} \cup\{t\}, Q\right)$. If $\mu_{1} \in \mathcal{M}_{A}^{s_{-t}}\left(S^{i^{\prime}} \cup\{t\}, Q\right)$, take $\bar{P} \in \mathcal{P}\left(\mu_{1}, S^{i^{\prime}}\right)$ as in expression (19). Therefore $U_{i}\left(\mu^{i^{\prime}}, P^{i^{\prime}}\right)=U_{i}\left(\mu_{1}, \bar{P}\right)$ for all $i \in S^{i^{\prime}}$ and we have

$$
\begin{aligned}
\sum_{i \in S^{*}} P_{\mu(i)}^{*} & =\sum_{i \in S^{i^{\prime}}} P_{\mu^{i^{\prime}}(i)}^{i^{\prime}}=v_{A}\left(S^{i^{\prime}} \cup\{0\}\right)-\sum_{i \in S^{i^{\prime}}} U_{i}\left(\mu^{i^{\prime}}, P^{i^{\prime}}\right) \\
& <v_{A}\left(\left(S^{i^{\prime}} \cup\{t\}\right) \cup\{0\}\right)-\sum_{i \in S^{i^{\prime}}} U_{i}\left(\mu_{1}, \bar{P}\right)=\sum_{i \in S^{i^{\prime}}} \bar{P}_{\mu_{1}(i)}+\sum_{j \in \mu_{1}(t)} a_{t j},
\end{aligned}
$$

where the first equality comes from condition $(b)$ of Proposition 5.1, the second one from Proposition A.2, in this Appendix, the strict inequality from the assumption and $U_{i}\left(\mu_{1}, P^{1}\right)=U_{i}\left(\mu^{i^{\prime}}, P^{i^{\prime}}\right)$ for all $i \in S^{i^{\prime}}$. This contradicts that $(s, \mathcal{O}(s))$ is a SPE because of condition (c) of Proposition 5.1. Therefore $\mu_{1} \notin \mathcal{M}_{A}^{s-t}\left(S^{i^{\prime}} \cup\{t\}, Q\right)$. 
By applying Lemma A.1, in this Appendix, to $S=S^{i^{\prime}}$ and $T^{\prime}=S^{i^{\prime}} \cup\{t\}$, there exists $\emptyset \neq T \varsubsetneqq T^{\prime}$, with $t \in T$, and $\mu^{\prime} \in \mathcal{M}_{A}^{s_{-t}}(T, Q)$ such that

$$
\sum_{i \in S^{i^{\prime}} \backslash T}\left(\sum_{j \in R(i)} a_{i j}-P_{R(i)}\right)>v_{A}\left(S^{i^{\prime}} \cup\{0\}\right)-v_{A}(T \cup\{0\}),
$$

where $\left(R(i), P_{R(i)}=s_{i}\right.$ for all $i \in S^{i^{\prime}} \backslash T$. Notice that

$$
\sum_{i \in S^{i^{\prime}} \backslash T} U_{i}\left(\mu^{i^{\prime}}, P^{i^{\prime}}\right)=\sum_{i \in S^{i^{\prime}} \backslash T}\left(\sum_{j \in R(i)} a_{i j}-P_{R(i)}\right)>v_{A}\left(S^{i^{\prime}} \cup\{0\}\right)-v_{A}(T \cup\{0\}) .
$$

Take $\mu^{\prime} \in \mathcal{M}_{A}^{s_{-t}}(T, Q)$ and $\bar{P}^{\prime} \in \mathcal{P}\left(\mu^{\prime}, T\right)$ as in expression (19). Therefore $U_{i}\left(\mu^{i^{\prime}}, P^{i^{\prime}}\right)=$ $U_{i}\left(\mu^{\prime}, \bar{P}^{\prime}\right)$ for each $i \in T \cap S^{i^{\prime}}$ and we have

$$
\begin{aligned}
\sum_{i \in S^{*}} P_{\mu(i)}^{*} & =\sum_{i \in S^{i^{\prime}}} P_{\mu^{i^{\prime}(i)}}^{i^{\prime}}=v_{A}\left(S^{i^{\prime}} \cup\{0\}\right)-\sum_{i \in S^{i^{\prime}}} U_{i}\left(\mu^{i^{\prime}}, P^{i^{\prime}}\right) \\
& <v_{A}(T \cup\{0\})-\sum_{i \in T \backslash\{t\}} U_{i}\left(\mu^{\prime}, \bar{P}^{\prime}\right)=\sum_{i \in T \backslash\{t\}} \bar{P}_{\mu^{\prime}(i)}^{\prime}+\sum_{j \in \mu^{\prime}(t)} a_{t j},
\end{aligned}
$$

where the first equality comes from condition (b) of Proposition 5.1, the second one from Proposition A.2 in this Appendix, the strict inequality from (35) and $U_{i}\left(\mu^{\prime}, \bar{P}^{\prime}\right)=$ $U_{i}\left(\mu^{i^{\prime}}, P^{i^{\prime}}\right)$ for all $i \in T$. This contradicts condition (c) of Proposition 5.1. Hence if $t \in M \backslash\left(S^{*} \cup S^{i^{\prime}}\right)$, then $v_{A}\left(S^{i^{\prime}} \cup\{0\}\right)=v_{A}\left(\left(S^{i^{\prime}} \cup\{t\}\right) \cup\{0\}\right)$.

Now, we prove $v_{A}\left(\left(\left(S^{*} \cup S^{i^{\prime}}\right) \backslash\left\{i^{\prime}\right\}\right) \cup\{0\}\right)=v_{A}\left(\left(M \backslash\left\{i^{\prime}\right\}\right) \cup\{0\}\right)$. Assume on the contrary that $v_{A}\left(\left(\left(S^{*} \cup S^{i^{\prime}}\right) \backslash\left\{i^{\prime}\right\}\right) \cup\{0\}\right)<v_{A}\left(\left(M \backslash\left\{i^{\prime}\right\}\right) \cup\{0\}\right)$. By monotonicity of $v_{A}$, let $\emptyset \neq T \subseteq M \backslash\left(S^{*} \cup S^{i^{\prime}}\right)$ be a minimal coalition (with respect to inclusion) such that

$$
v_{A}\left(\left(\left(S^{*} \cup S^{i^{\prime}} \cup T\right) \backslash\left\{i^{\prime}\right\}\right) \cup\{0\}\right)>v_{A}\left(\left(\left(S^{*} \cup S^{i^{\prime}}\right) \backslash\left\{i^{\prime}\right\}\right) \cup\{0\}\right) .
$$

Then, there is some $t \in T$ such that,

$$
v_{A}\left(\left(\left(S^{*} \cup S^{i^{\prime}} \cup T\right) \backslash\left\{i^{\prime}\right\}\right) \cup\{0\}\right)>v_{A}\left(\left(\left(S^{*} \cup S^{i^{\prime}} \cup T\right) \backslash\left\{i^{\prime}, t\right\}\right) \cup\{0\}\right) .
$$

Notwithstanding, since $v_{A}\left(\left(S^{i^{\prime}} \cup\{i\}\right) \cup\{0\}\right)=v_{A}\left(S^{i^{\prime}} \cup\{0\}\right)$ for all $i \in M \backslash\left(S^{*} \cup S^{i^{\prime}}\right)$

$$
\begin{aligned}
0=v_{A}\left(\left(S^{i^{\prime}}\right.\right. & \cup\{t\}) \cup\{0\})-v_{A}\left(S^{i^{\prime}} \cup\{0\}\right) \\
& \geq v_{A}\left(\left(\left(S^{*} \cup S^{i^{\prime}} \cup T\right) \backslash\left\{i^{\prime}\right\}\right) \cup\{0\}\right)-v_{A}\left(\left(\left(S^{*} \cup S^{i^{\prime}} \cup T\right) \backslash\left\{i^{\prime}, t\right\}\right) \cup\{0\}\right)>0,
\end{aligned}
$$

where the inequality comes from the buyers-submodular condition and the strict inequality from (36). This is a contradiction. Hence, $v_{A}\left(\left(\left(S^{*} \cup S^{i^{\prime}}\right) \backslash\left\{i^{\prime}\right\}\right) \cup\{0\}\right)=$ $v_{A}\left(\left(M \backslash\left\{i^{\prime}\right\}\right) \cup\{0\}\right)$. 


\section{References}

[1] Arribillaga, R., Massó, J. and Neme, A. (2013). On the Structure of Cooperative and Competitive Solutions for a Generalized Assignment Game. Barcelona GSE Working Paper Series, Working Paper No. 740, Barcelona.

[2] Ausubel, L. and Milgrom, P. (2002). Ascending auctions with package bidding. Frontiers of Theoretical Economics 1(1), Article 1.

[3] Camiña, E. (2006). A generalized assignment game. Mathematical Social Sciences $52: 152-161$.

[4] Demange, G., Gale, D. and Sotomayor, M. (1985). Multi-item auctions. Journal of Political Economics 94:863-872.

[5] Gale, D. (1960). The theory of linear economic models, Mc Graw Hill, New York.

[6] Jaume, D., Massó, J. and Neme, A. (2012). The multiple-partners assignment game with heterogeneous sales and multi-unit demands: competitive equilibria. Mathematical Methods of Operations Research 76:161-187.

[7] Kaneko, M. (1976). On the core and competitive equilibria of a market with indivisible goods. Naval Research Logistic Quarterly 23:321-337.

[8] Pérez-Castrillo, D. and Sotomayor, M. (2001). A simple selling and buying procedure. Journal of Economic Theory 103:461-474.

[9] Shapley, L. (1962). Complements and substitutes in the optimal assignment problem. Naval Research Logistics Quarterly 9:45-48.

[10] Shapley, L. and Shubik, M. (1972). The assignment game I: The core, International Journal of Game Theory 1:111-130.

[11] Sotomayor, M. (1992). The multiple partners game. In: Majumdar M. (ed.), Equilibrium and dynamics: Essays in Honor to David Gale. Macmillan, pp. 322-336.

[12] Sotomayor, M. (2002). A labor market with heterogeneous firms and workers. International Journal of Game Theory 31:269-283.

[13] Sotomayor, M. (2007). Connecting the cooperative and competitive structures of the multiple-partners assignment game. Journal of Economic Theory 134:155-174.

[14] Sotomayor, M. (2013). Correlating new cooperative and competitive concepts in the time-sharing assignment game. FEA-USP Working Paper Series 2013-02, São Paulo.

[15] Tauman, Y., Urbano, A. and Watanabe, J. (1997). A model of multiproduct price competition. Journal of Economic Theory 64:416-424. 
[16] Thompson, G. (1980). Computing the core of a market game. Extremal methods and systems analysis. A.V. Fiacco and K.O. Kortanek (eds.). Lecture Notes in Economics and Mathematical Systems no. 174. Springer, pp. 312-334.

[17] Vickrey, W. (1961). Counterspeculation, auctions and competitive sealed tenders. Journal of Finance 16:8-37. 